\title{
Breaking size-segregation waves and mobility feedback in dense granular avalanches
}

DOI:

10.1007/s10035-018-0818-x

\section{Document Version}

Accepted author manuscript

Link to publication record in Manchester Research Explorer

\section{Citation for published version (APA):}

Vaart, K. V. D., Thornton, A. R., Johnson, C., Weinhart, T., Jing, L., Gajjar, P., ... Ancey, C. (2018). Breaking sizesegregation waves and mobility feedback in dense granular avalanches. Granular Matter, 20(46). https://doi.org/10.1007/s10035-018-0818-x

\section{Published in:}

Granular Matter

\section{Citing this paper}

Please note that where the full-text provided on Manchester Research Explorer is the Author Accepted Manuscript or Proof version this may differ from the final Published version. If citing, it is advised that you check and use the publisher's definitive version.

\section{General rights}

Copyright and moral rights for the publications made accessible in the Research Explorer are retained by the authors and/or other copyright owners and it is a condition of accessing publications that users recognise and abide by the legal requirements associated with these rights.

\section{Takedown policy}

If you believe that this document breaches copyright please refer to the University of Manchester's Takedown Procedures [http://man.ac.uk/04Y6Bo] or contact uml.scholarlycommunications@manchester.ac.uk providing relevant details, so we can investigate your claim.

\section{OPEN ACCESS}




\title{
Breaking size-segregation waves and mobility feedback in dense granular avalanches
}

\author{
K. van der Vaart • A.R. Thornton - C.G. Johnson • \\ T. Weinhart • L. Jing · P. Gajjar • J.M.N.T. Gray · C. Ancey
}

Received: date / Accepted: date

\begin{abstract}
Through experiments and discrete particle method (DPM) simulations we present evidence for the existence of a recirculating structure, that exists near the front of dense granular avalanches, and is known as a breaking sizesegregation (BSS) wave. This is achieved through the study of three-dimensional bidisperse granular flows in a moving-bed channel. Particle-size segregation gives rise to the formation of a largeparticle-rich front and a small-particle-rich tail with a BSS wave positioned between the tail and front. We experimentally resolve the structure of the BSS wave using refractive-index matched
\end{abstract}

K. van der Vaart

Civil and Environmental Engineering, Stanford University, Stanford, CA 94305, U.S.

A. R. Thornton · T. Weinhart

Multiscale Mechanics, ET/MESA+, University of Twente, P.O. 217, 7500AE Enschede, The Netherlands

L. Jing

Department of Civil Engineering, The University of Hong Kong, Pokfulam Road, Hong Kong, China

P. Gajjar

Henry Mosely X-Ray Imaging Facility, School of Materials, University of Manchester, Manchester M13 9PL, United Kingdom

C. G. Johnson · J. M. N. T. Gray

School of Mathematics and Manchester centre for Nonlinear Dynamics, University of Manchester, Manchester M13 9PL, United Kingdom

C. Ancey

Environmental Hydraulics Laboratory, École Polytechnique Fédérale de Lausanne, Écublens, 1015 Lausanne, Switzerland scanning and find that it is qualitatively similar to the structure observed in DPM simulations. Our analysis demonstrates a relation between the concentration of small particles in the flow and the amount of basal slip, in which the structure of the BSS wave plays a key role. This leads to a feedback between the mean bulk flow velocity and the process of particle-size segregation. Ultimately, these findings shed new light on the recirculation of large and small grains near avalanche fronts and the effects of this behaviour on the mobility of the bulk flow.

Keywords Avalanches · Size-segregation . Mobility feedback · Basal slip · Moving-bed channel

\section{Introduction}

Naturally occurring granular flows, such as debris flows, snow avalanches, and pyroclastic flows, usually exhibit bouldery or large-particle-rich fronts, as is evident from their deposits [1-10]. Such largeparticle fronts are known to interact with the more mobile flow behind, inducing behaviours such as fingering [11-15] or the formation of lateral levees [16-21]. Fig. 1 shows an example of these phenomena in a snow avalanche deposit. Up close it can be seen that large ice and snow boulders make up the front of the deposit, while zooming out shows lateral levees and fingers.

Both lab-scale and large outdoor experiments $[12,13,19,21-24]$ have demonstrated that the 

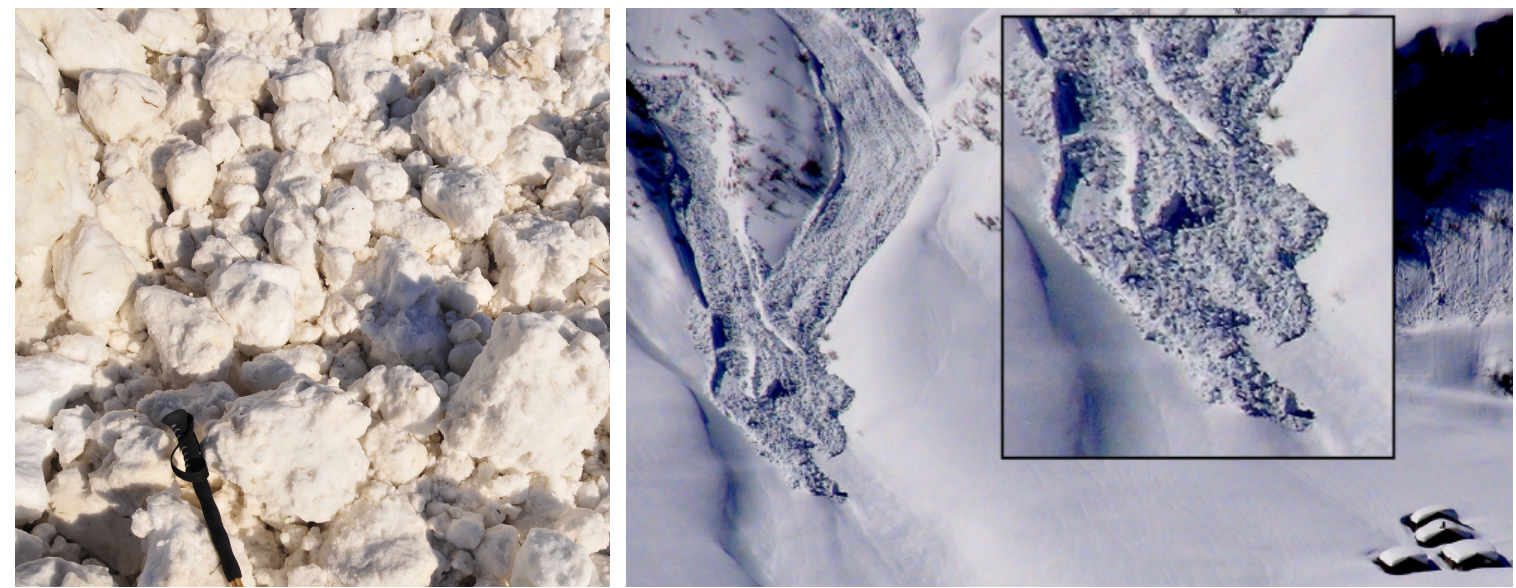

Fig. 1 Left: An example of a large-particle front in a snow avalanche deposit. The black object is the handle of a ski pole, placed for scale comparison. Right: An example of lateral levees and fingering in snow avalanche deposits in the Alps. The avalanche has split up in a number of fingers which are bounded by lateral levees. The inset shows a zoom-in.

process of particle-size segregation - the spatial separation of different-sized grains during flow $[25$, 26 - plays a key role in the formation of largeparticle fronts, lateral levees and fingers. Namely, in dense gravity-driven flows particle-size segregation causes large particles to rise to the free surface of the flow while causing small particles to sink to the bed [e.g. 27-36]. The surface layers of the flow typically have the highest velocities and so the segregated larger particles are transported to the front of the flow, while small particles are left behind in the avalanche tail. Coarse material in the front that is deposited on the bed and overrun by the advancing flow can subsequently be re-entrained, segregate and travel back to the front once again.

The recirculation of grains and spatial distribution of different sized particles in avalanche fronts caused by size segregation was described theoretically by Thornton and Gray [37], who termed the structure a breaking size-segregation (BSS) wave. This BSS wave occurs near the front of a propagating flow [12,38-40], and is the mechanism by which a large-particle front interacts with the finer and more mobile particles behind. Large particles may recirculate many times in a BSS wave at the front of a flow, while continually being advected away from the flow centre-line, toward the sides of the flow, and are eventually deposited in a lateral levee [21]. Thus, the BSS wave is central to the formation of large-particle fronts and lateral levees.

One important reason for the scientific interest in large-particle fronts, lateral levees and particle-size segregation, is that these effects play a critical role in granular flows because of their feedback on the mobility of the bulk [14, 24, 39, 41, 42]. This so-called 'mobility feedback' affects the avalanche run-out distance, which is a critical parameter to know for hazard mitigation. For example, lateral levees are known to increase avalanche run-out distance because they channelize the flow. The inside of levees can be lined by a layer of deposited fine grains, which reduces the bulk friction both in laboratory and experiments and natural debris and pyroclastic flows [43]. This levee morphology arises through segregation in the BSS wave $[12,43]$.

Although a vast amount of literature exists on granular flows in nature, the precise details and mechanisms underlying the feedback between bulk mobility and size-segregation is still an outstanding problem. If we want to accurately predict the behaviour of avalanches and debris flows it is of the utmost importance to understand how sizesegregation and mobility feedback are related. A detailed understanding of the BSS wave can also inform interpretation of deposits from natural flows. The structure of the BSS wave determines the sedimentology of a flow deposit near the distal termination, and analysis of the deposit 


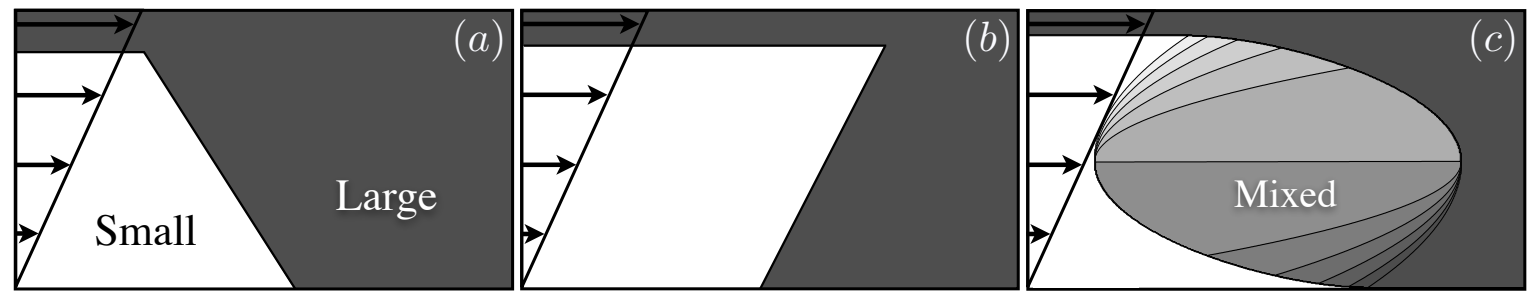

Fig. 2 Schematic of the formation of a breaking size-segregation wave in a granular flow with a linear velocity profile, as indicated by the arrows. The white region corresponds to a pure small-particle phase, while the dark grey region corresponds to a pure large particle phase. Grey-scales in between indicate a mixing of the two species. (a) A large-particle front is formed when a segregated flow transports large particles to the front. This transportation is the result of the faster moving free-surface layers of the flow. (b) The small-particle tail is sheared over the front. Particle-size segregation makes this an unstable situation. (c) As large particles begin to rise and small particles sink, the "wave" collapses, and a complex recirculating structure is formed: a breaking size-segregation wave. The wave is characterised by an oval-like "lens" region that separates the small-particle tail and the large-particle front.

sedimentology can allow the size of the BSS wave to be inferred, with consequent constraints on the rate of segregation [21].

The aim of this work is to visualise the interior of gravity driven granular flows that exhibit a large-particle-rich front and a small-particlerich tail, and to study their internal structure. In doing so we aim to verify theoretical predictions (which will be discussed below) for the internal structure of these flows and understand how particle-size segregation affects the bulk flow behaviour.

\section{Breaking Size-segregation Waves}

The size-segregation model for dense granular flows by Gray and Thornton [44] has allowed for the theoretical exploration of size-segregation near avalanche fronts $[37,39,40,42]$. In one of these works, Thornton and Gray [37] showed that a complex recirculating structure can exist between the large-particle front and small-particle tail. They called this structure a breaking size-segregation (BSS) wave after the wave breaking of water waves when approaching a shoreline. Before the study of Thornton and Gray [37] was published, a recirculation of large particles near avalanche fronts had already been observed in several experimental studies [12, 13, 19], however the actual structure of the recirculating zone had eluded investigators for reasons that will be explained in Section 2.1.

Fig. 2 shows a schematic of how a BSS wave is thought to be formed: When small particles are sheared over the large particles that have accumulated at the front of the avalanche, the resulting configuration is unstable as a consequence of size segregation. Size segregation will cause the small particles to sink and the large particles to rise. This causes the 'wave' of small particles to break and form a so-called 'lens' region where small particles sink and large particles rise. This lens, which travels behind the front separating it from the small-particle tail, is the BSS wave. Thornton and Gray [37] predict that the length of a BSS wave scales inversely with the strength of segregation. As such, the length can be many times the flow depth if segregation is weak.

Recently, Marks et al. [45] reported on experiments with a single large intruder flowing in a 2D granular avalanche on a moving-bed channel. They demonstrated that the large intruder recirculates in a region in the front half of the flow, reminiscent of a BSS wave. Their theoretical model was able to accurately predict the average position of this intruder in the flow.

Gajjar et al. [46] presented the theoretical background for the study presented here and included preliminary experimental and simulation data as a proof-of-concept and for theoretical comparison. Their main contribution was to extend the current theory on BSS waves [37] to include the effect of size-segregation asymmetry [47, 48]. Here we provide a detailed study of the experimental and simulation results and the first direct evidence for the existence of BSS waves in bidisperse flows. 


\subsection{Difficulty of Studying BSS Waves}

An important question that arose following the theoretical study of BSS waves is what role its structure plays for the bulk flow behaviour. In other words, how is size segregation coupled to the bulk flow through the recirculating structure of a BSS wave? In order to answer this question an experimental verification and a detailed study of BSS waves is required. Unfortunately this has proven challenging because of side-wall effects and the complex time-dependence of BSS waves. Firstly, in any geometry that is used for the study of size-segregation, the side-walls, through which imaging is done, are predominantly occupied by smaller particles [30, 49]. This obscures the real structure of the BSS wave. Secondly, the challenge of the time-dependent nature of BSS waves lies in the fact that it takes a long time for one to develop. When it has formed it travels close to the front of the flow, but not necessarily at the same velocity. The reason for this is that if more large particles are carried to the front than are deposited on the bed, the largeparticle front grows, thereby pushing the BSS wave back [39, 40]. Thus, in order to study BSS waves, a very long chute is required to allow a steady BSS wave to emerge. A moving camera is then needed to somehow track and capture the motion of the BSS wave.

The above discussed problems can be avoided by studying avalanche deposits, as was done successfully by Johnson et al. [21], who presented the first indirect evidence for the existence of BSS waves in large-scale debris-flow experiments. However, in order to capture the structure of BSS waves directly and in real-time, a different setup is required.

\subsection{The Moving-Bed Channel}

We have developed an effective experimental setup that allows for the visualisation and study of the structure and motion of BSS waves. It consists of a combination of two existing techniques. Namely: a moving-bed channel [45, 50-55] and the noninvasive imaging technique Refractive Index Matched Scanning (RIMS) [56, 57], that allows for the visualisation of the interior of a granular flow.
A moving-bed channel is similar to an ordinary inclined channel or chute, with the exception that the bed moves upstream at a specific velocity. This drags the lower layers of the flow upstream, while the upper layers move downstream under gravity. This significantly decreases the required length of the channel and circumvents the problem of the time-dependence of BSS waves, since the flow can continue indefinitely and reach a steady-state. Because the flow and the BSS wave are stationary in the reference frame of the lab, RIMS can be used to visualise the interior of the flow, far from the side-wall, thus circumventing the problem of the preferential position of small particles near the side-walls.

In addition to experiments, we have implemented the moving-bed channel geometry in discrete particle method (DPM) simulations. Although there are no problems of visualising the interior of a granular flow in the DPM simulations, the moving-bed channel is required to solve the timedependence problem: A normal periodic chute flow - the most commonly used geometry for studying gravity driven flows - does not allow the formation of a large-particle front, whereas the movingbed channel does.

\section{Methods}

Three-dimensional (3D) experiments and simulations have been performed in a moving-bed channel $[45,50,54,55,58]$ with bidisperse granular mixtures. We first performed the experiments in order to establish the existence of BSS waves. Next, we performed the simulations with the aim to create a better system, using a smaller particlesize ratio between the two species, as well as a longer channel and periodic boundary conditions for the side-walls (perpendicular to the flow direction). Our goal of improving on the experiment in the simulations is the reason we have used a different parameter set for the simulations compared to the experiment. The reasons for this choice are explained in more detail in Sec. 4.2. This difference in parameters prohibits us from doing a quantitative comparison between experiment and simulation. Note that even without this choice of different parameters, a quantitative comparison is prevented by the fact that in the 
experiment there is an interstitial fluid, whereas there is none in the simulations.

In the next section we discuss the general methodologies, followed by the experimental and simulation methods in detail in Sec. 3.2 and Sec. 3.3.

\subsection{General Methodology}

In a moving-bed channel a continuous flow is created by placing a granular mixture on an inclined channel, with inclination angle $\theta$, where the bed is a conveyor belt. A schematic of the experimental conveyor belt is shown in Fig. 3. In all cases our coordinate system, as shown in Fig. 3, is such that $x$ points in the downstream direction, $y$ in the cross-stream (transversal) direction, and $z$ perpendicular to the bed. Corresponding velocities of the flow in those directions are $u, v$ and $w$, respectively. The origin lies at the bottom of the upstream (left) wall. The conveyor belt moves with a negative velocity $-u_{b}$, i.e. it moves in the upstream direction, where we define $u_{b}>0$. It drags the bottom of the mixture upstream, while the top of the mixture avalanches downstream. The flowing mixture is prevented from moving out of the channel at the upstream and downstream ends by walls (simulation) or gates (experiment). In the experiment the channel has two side-walls, while in the DPM simulations the system is periodic in the $y$-direction.

All experiments and simulations are performed using size-bidisperse mixtures consisting of equal density small and large particles with diameters $d_{s}$ and $d_{l}$, respectively. Both the channel dimensions and particle dimensions differ between the simulations and experiments for reasons that will be explained in Sec. 4.2. We define a global volume (or solids) fraction of small particles $\Phi=$ $V_{s}^{p} /\left(V_{s}^{p}+V_{l}^{p}\right)$, where $V_{s}^{p}$ and $V_{l}^{p}$ are the total volumes of small and large particles in the mixture, respectively.

Using coarse-graining (CG) [59-61] on the particle positions and velocities - after a steady state has been reached - we obtain time and widthaveraged 2D continuum fields for the downstream velocity $u$, the local small particle volume fraction $\phi_{s}$, and the bulk friction $\mu=\left|\sigma_{x z}\right| /\left|\sigma_{z z}\right|$ [62]. Here $\sigma_{x z}$ and $\sigma_{z z}$ are the shear stress and downward normal stress, respectively. The local vol- ume fraction is defined such that the void space is ignored, i.e., the large-particle volume fraction $\phi_{l}$ and the small-particle volume fraction $\phi_{s}$ sum to unity: $\phi_{s}+\phi_{l}=1$. For simplicity, and because it is the terminology in previous works, from here on we will refer to $\phi_{s}$ simply as $\phi$ and to $\phi_{l}$ as $1-\phi$. We use a CG-width of $\hat{w}=0.5=w / d_{m}$ for all simulations and experiments, where $d_{m}=$ $\Phi d_{s}+(1-\Phi) d_{l}$ is the mean particle diameter and $w$ is the width of the CG kernel [60]. The CG-width is chosen such that the obtained fields are independent of it, as described by Tunuguntla et al. [60]. The time-averaging CG-width $w_{t}$ is the length of the entire time-window in which data has been acquired - as the flow is in steady-state.

Depending on the inclination angle $\theta$ of the moving-bed channel and the belt speed, the flow depth along the length of the channel varies: it can either be a uniform depth; a deeper flow at larger $x$; or a shallower flow at larger $x$. For this study we aimed to keep the inclination angle and belt speed at values that resulted in a uniform depth along the length of the channel. This flow mimics a region of a free-surface flow that is located just behind the avalanche front. Thus, the actual tail of the avalanche and the front, where the flow depth decreases to zero, do not exist in this configuration. We refer the reader to the the-

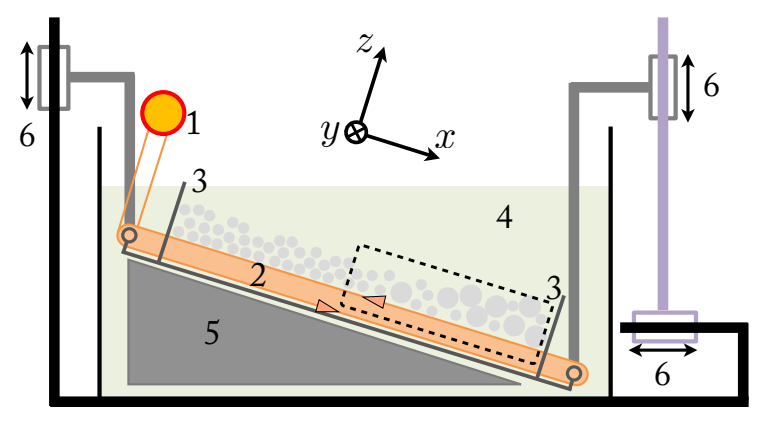

Fig. 3 A schematic of the experimental setup, a moving-bed channel submerged in an aquarium. The dashed line indicates the imaging window. The numbers indicate: (1) the motor; (2) the conveyor belt; (3) the upstream and downstream gates that keep the granular mixture in the channel, while allowing the conveyor belt to pass; (4) the aquarium filled with RIMS liquid (green); (5) a plastic box with the purpose of decreasing the total volume of RIMS liquid required to fill the aquarium; (6) The inclination of the belt is changed by sliding along these points. Image courtesy of J.-L. Pfister. 
oretical companion paper [46] for details on the theoretical validity of this approach. In the experiments we fine-tuned the belt speed and inclination angle by hand while the mixture was flowing. As a result of the shallow flow depth in terms of particle diameters, we were able to get a uniform flow depth with an error not less than $5 \%$ to $20 \%$ (see e.g. Fig. 5). In order to reduce this error a deeper flow would have been required, but unfortunately further increasing the flow depth in the experiment proved impractical as explained in Sec. 4.2. For the simulations we automated the fine tuning of the belt speed by adjusting it based on the position of centre-of-mass of the flow, as explained in more detail in Sec. 3.3. For intermediate values of $\Phi_{s}$ (between $20 \%$ and $70 \%$ ) this approach did not give rise to a uniform depth along the length of the channel, but instead resulted in a deeper flow at the upstream and down stream ends (see Sec. 4.5).

\subsection{Experimental methods}

In the experiments the diameters of the large and small particles are $d_{l}=14 \mathrm{~mm}$ and $d_{s}=$ $5 \mathrm{~mm}$, respectively. The particle material properties are the same for both species and are discussed in Sec. 3.2.1. Fig. 3 shows a schematic of the experimental setup. The moving-bed channel is submerged in an aquarium in order to perform Refractive Index Matched Scanning (RIMS) [56, 57], as will be explained in Sec. 3.2.1. Gates are positioned at the top and bottom ends of the channel. The belt moves underneath these gates while the granular material is blocked. The channel length is $208 d_{s}$ and its width is $20 d_{s}$. One of the side-walls is transparent to allow imaging. The conveyor-belt is entirely made of metal, so that it is resistant to the corrosive RIMS fluid. To add roughness to the belt, rectangular metal bars of $0.2 d_{s}$ height and $0.4 d_{s}$ width, are soldered transversally on the belt at $1.2 d_{s}$ intervals. The mean flow height is $\bar{h}=10 d_{s}$, while $\Phi=70 \%$. We use a belt speed $u_{b}=14.4 d_{s} s^{-1}$ and an inclination angle $\theta=19.8^{\circ}$. The experiment is prepared by pouring in the mixture of grains, after which the angle and belt speed are varied until a uniform depth along the length of the channel is obtained. During this process the BSS wave forms and becomes steady within a couple of minutes, after which data gathering can start.

\subsubsection{RIMS Implementation}

The motion of the particles in the experiment is revealed using RIMS [56, 57]. Only the particle motion on the stream-wise centre-line is captured because scanning the entire width would require stopping the flow. We image only part of the downstream end of the channel where the BSS wave forms, as indicated in Fig. 3.

The particles used are made of borosilicate glass with a density of $\rho_{p}=2230 \mathrm{~kg} \mathrm{~m}^{-3}$ and a refractive index $n_{p}=1.473$. The index-matched liquid for RIMS is a 61:39 $( \pm 0.3)$ mixture by volume of benzyl-alcohol and ethanol. This results in a RIMS liquid with viscosity $\mu_{R}=3 \mathrm{mPas}$ and density $\rho_{R}=995 \mathrm{~kg} \mathrm{~m}^{-3}$. The refractive index of the RIMS liquid $n_{f}$ is matched as closely as possible to $n_{p}$, with a precision of $\mathcal{O}\left(10^{-3}\right)$. In order to maintain an accurate matching between $n_{f}$ and $n_{p}$ the temperate of the room is controlled and held at $18 \pm 0.2^{\circ} \mathrm{C}$.

The chosen combination of RIMS fluid and borosilicate glass beads has the advantage of a low viscosity fluid and a large density ratio between the fluid and particles. This ensures that the Stokes number, which describes the characteristic response time of the fluid divided by the characteristic response time of the particle $[63$, 64], is much bigger than one. Therefore the fluid drag forces are small compared to the gravitational and shearing forces on the particles and the flow behaviour is close to dry flows.

A small amount of Rhodamine $6 \mathrm{G}$ is added to the RIMS liquid as a fluorescent dye. Rhodamine $6 \mathrm{G}$ has a peak excitation wavelength at $528 \mathrm{~nm}$ and peak emission wavelength at $553 \mathrm{~nm}$. Two laser sheets are created with a 4 watts diodepumped solid state laser with a wavelength of $532 \mathrm{~nm}$, using a combination of two convex lenses, a beam splitter and two rod lenses. The two sheets are needed to span a large enough area of the flow. The entire channel is submerged in an aquarium filled with RIMS liquid. A Basler A406k camera, at a frame rate of $40 \mathrm{~Hz}$, is used to image the flow through the glass side-wall of the channel. Fig. 4 shows a photograph of the experimental 


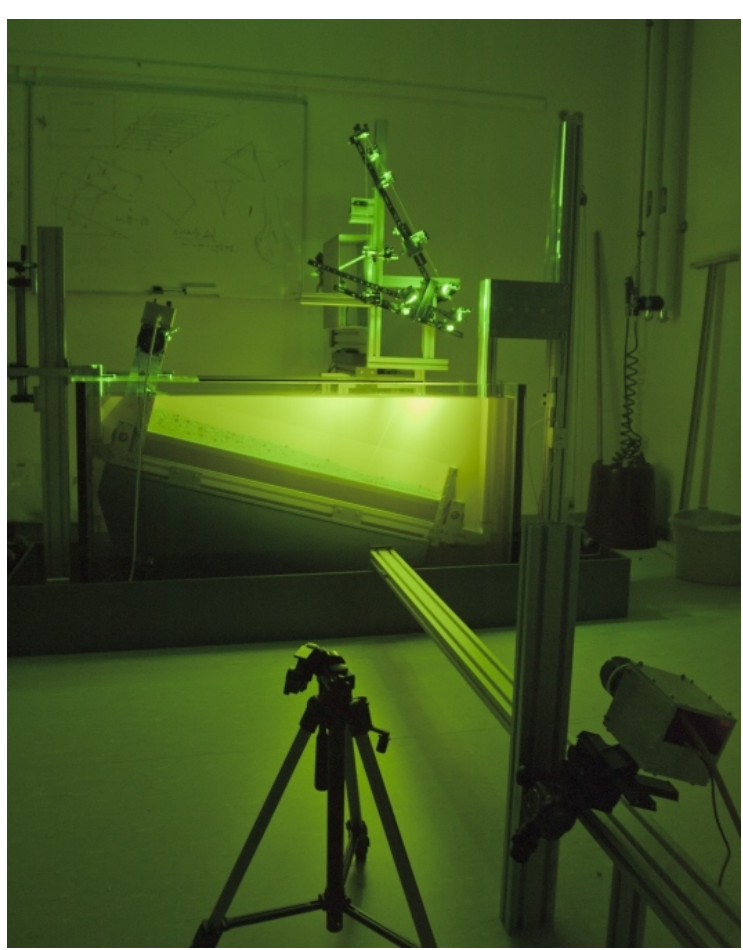

Fig. 4 A photograph of the experimental setup. The dual-laser can be seen above the tank. See Fig. 3 for details on different parts of the setup.

setup with the dual-laser setup positioned above the tank.

The particle cross-sections obtained on the centre-line of the channel are tracked over time, with the minimum and maximum diameters used to determine whether that cross-section corresponds to a small or large particle. The large size ratio between the grains minimizes identification errors. If a cross-section disappears and the diameter has not been conclusively determined it is ignored. This occurs when the recorded diameter of the tracked cross-section lies between $d_{s}$ and $\frac{4}{5} d_{s}$ and the time of tracking it is less than 3 seconds. This cutoff is based on the high probability that a small particle moves perpendicular to the flow direction within the interval of a couple of seconds. If a cross-section is tracked for this short time-interval and stays in this diameter range there is a possibility that it is a large particle. If a diameter below $\frac{4}{5} d_{s}$ is detected it is identified as a small particle because if a large particle intersects the laser sheet close to its edge (in order to give rise to a cross-section with a di- ameter small than $\frac{4}{5} d_{s}$ ) it results in a disk on the raw data image that is relatively blurry. The particle identification algorithm cannot resolve these blurry cross-sections. In practice roughly $5 \%$ of disks are ignored in this way. For particle identification we use a combination of image convolution and least-squares fitting [65] and tracking of particles is done via a Voronoï-based method [66].

\subsection{Particle Simulations}

Three-dimensional discrete particle method (DPM) simulations of size-bidisperse mixtures of dry frictional spherical particles are performed using MercuryDPM [MercuryDPM.org ; 67, 68]. All simulation parameters are non-dimensionalised such that the small-particle diameter is $d_{s}=1.0$, the particle density is $\rho_{p}=6 / \pi$, the mass $m=1$, and the gravitational acceleration is $g=1$. The diameter of the large particles is $d_{l}=2.4$. This results in a smaller size ratio $S=d_{l} / d_{s}=2.4$ compared to $S=2.8$ in the experiments. The simulations are conducted in a box of length $300 d_{s}$ with fixed end walls, and width $8.9 d_{s}$. This channel is longer compared to the experimental channel, which has a non-dimensional length of $208 d_{s}$. The side-walls are periodic in order to remove sidewall effects. The channel inclination angle $\theta=$ $24^{\circ}$. The global small-particle volume fraction $\Phi$ is varied between 0 and $100 \%$, while keeping the total mixture volume constant. We calculate the flow depth $h(x)$ by extrapolating $\sigma_{z z}(x, z)$ to zero, as described in Eq. (42) in [62]

A linear spring-dashpot model $[59,69]$ with linear elastic and linear dissipative contributions is used for the normal and tangential forces between particles. The normal spring and damping constants are $k_{n}=2 \times 10^{5} \mathrm{mg} / d_{s}$ and $\gamma_{n}=$ $50 \sqrt{g / d_{s}}$. The tangential spring and damping constants are $k_{t}=(2 / 7) k_{n}$ and $\gamma_{t}=\gamma_{n}$, such that the frequency of normal and tangential contact oscillation and the normal and tangential dissipation are equal. The tangential force also truncates so that it is always less than $1 / 2$ of the normal force. The restitution coefficient for collisions $r_{c}=0.1$ and the contact duration $t_{c}=0.005$. The restitution coefficient used is less than the typical known values for glass $(\sim 0.9)$ [70]. Specifying $r_{c}$ and $t_{c}$ results in a different stiffness depending 
on the particle size. We verified that our findings are not the result of this difference in stiffness nor the dependence on $r_{c}$ and $t_{c}$. The inter-particle friction is chosen to be $\mu_{p}=0.5$, which is close to the measured values for glass [71].

All simulations make use of a rough bed composed of randomly positioned particles with diameter $d_{b}=1.7$, which are rigidly attached to the moving bed, moving upstream with velocity $-u_{b}$ in the upstream direction. In order to correctly model the relative velocities between the bed and the flowing particles, the bed particles are endowed with the velocity $-u_{b}$. The typical range of velocities $u_{b}$ used is between $1.0 \sqrt{g d_{s}}$ and $4.5 \sqrt{g d_{s}}$. This approach is an improvement over the one used to obtain the preliminary data shown in the companion paper [46], where the bed particles, despite moving, had an internal velocity of zero. Hence the energy dissipation of collisions between flow and base particles was incorrectly calculated as the velocity of the bed particles was not included when computing the relative velocity. This error has a negligible effect on the segregation profile but does effect the velocity profile.

Before the gathering of data begins, the uniform flow depth is obtained by automatically updating the belt speed based on the instantaneous distance of the horizontal centre-of-mass of the mixture $\langle x\rangle$ to the centre of the channel $x_{c}=150$. The acceleration provided to the bed is $\Delta a=$ $\left(\langle x\rangle-x_{c}\right) / 2000$ and is calculated and applied every 5000 time-steps. This ensures a very gradual approach to a homogenous flow-depth with little disturbance to the flow. Once the fluctuations in the belt speed become negligible, i.e., when $|\Delta a|<0.005$ for more than $2.5 \cdot 10^{4}$ time-steps, the acquisition of data begins. The velocity control is maintained during data gathering. Details of the bed creation process can be found in [72], whereas a detailed description of different bed types and their effect on the macroscopic friction experienced by the flow can be found in [73].

A small inclined wall is placed between the base and the vertical upstream wall in order to prevent small particles being squeezed between the wall and particles of the bed. This wall intersects with the upstream wall at $(0,1.5)$ and with the $z=0$ plane at $(7,0)$. It guides small particles away from the bed before they impact the upstream wall. This improves the dynamics close to the wall by preventing extreme forces on particles that result from the bed pushing them into the wall. Because this inclined wall is far away from the centre of the channel it does not affect the BSS wave.

\section{Results}

\subsection{Phenomenology and Structure}

As a result of particle-size segregation large particles accumulate at the downstream end of the channel and small particles at the upstream end. We refer to these two regions of pure large particles and pure small particles as the large-particle front and the small-particle tail, respectively. Separating the front and tail is a breaking size-segregation (BSS) wave where both species exist together [37, 46]. Fig. 5(a) and $5(b)$ show snapshots of the flow in the experiment and simulation, in which the large-particle front, the BSS wave, and the smallparticle tail are visible. Note that for the experiments only a section of the small-particle tail is shown because we have not imaged the full channel, due to experimental challenges. In the BSS wave segregation recirculates the large and small particles: Small particles that travel to the front of the flow segregate to the bed, whereas large particles that have been deposited at the bed are re-entrained and segregate to the free surface. There are two notable features, firstly, the smallparticle tail being typically several times longer than the large-particle front, and secondly, the length of the BSS wave, which in both the experiment and simulation is several times the flow depth. Johnson et al. [21] estimated the length of a BSS wave in their large-scale experimental debris flows on the order of around $3 \mathrm{~m}$, with a flow depth of $0.2 \mathrm{~m}$. The particle diameter in these experiments ranged from 0.06 to $32 \mathrm{~mm}$.

Fig. 5(c) and $5(d)$ show the time and widthaveraged small-particle volume fraction fields $\phi(x, z)$ for the experiment and simulation shown in the snapshots. The $\phi(x, z)$-fields show more clearly the structure of the BSS wave. The BSS wave in the experiment and simulation are qualitatively similar. They both exhibit a characteristic 'tail' of large particles that extends into the bulk of the 

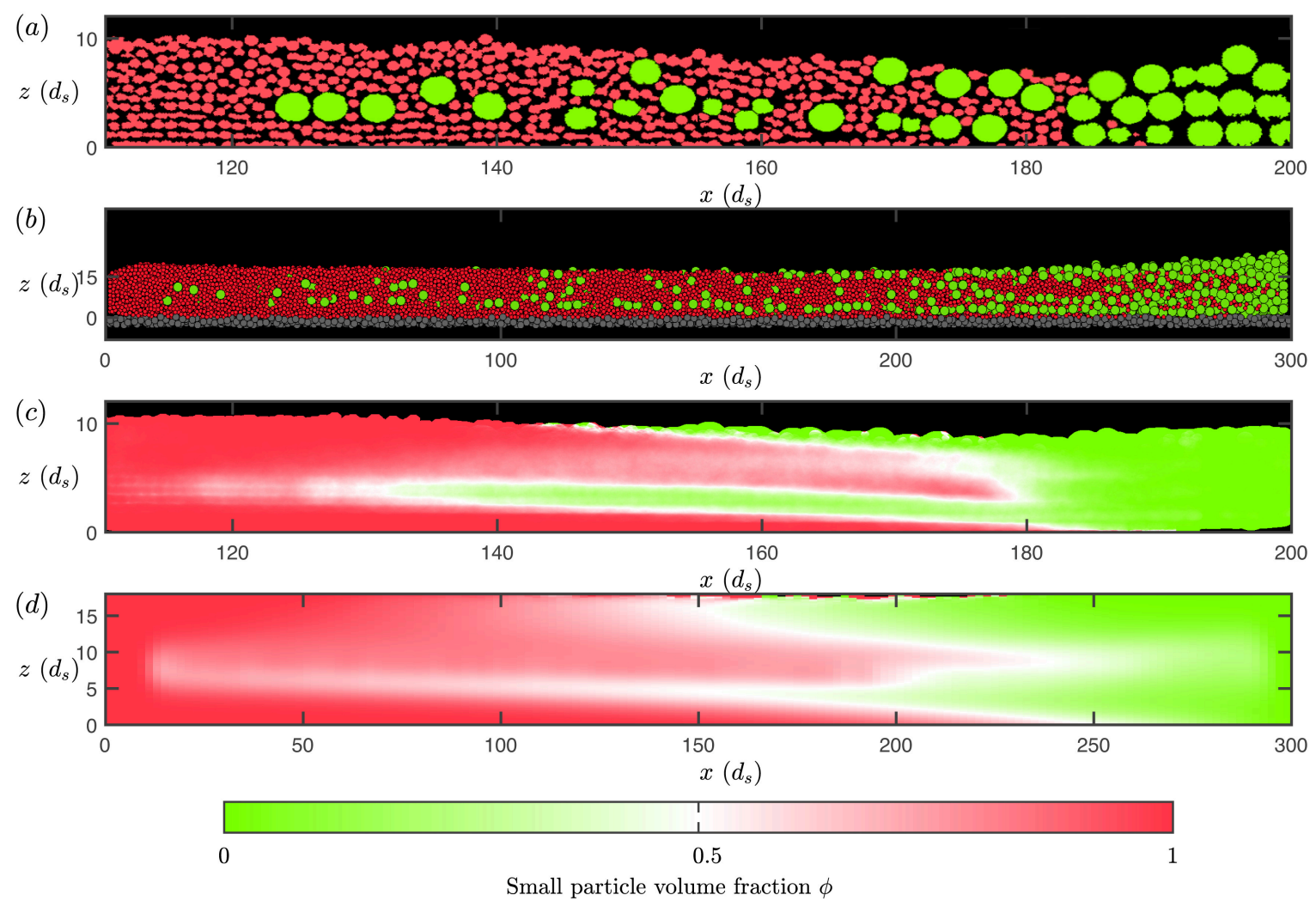

Fig. 5 (a) Snapshot of the interior of the flow in the experiment $(\Phi=70 \%)$; captured using RIMS. Reprinted with permission from [46]. (b) Snapshot of a simulation $(\Phi=70 \%)$. The fixed base particles are shown in grey. (c) The experimental time-averaged small-particle volume fraction $\phi(x, z)$. Reprinted with permission from [46]. (d) The time-averaged small-particle volume fraction field for the simulations. Note that the downstream wall in the experiment is at $x=208$, while it is at $x=300$ in the simulation. The upstream wall is at $x=0$. Because of the difference in flow and particle parameters between the experiment and simulation, only a qualitative comparison is possible here.

upstream small-particle-rich region of the flow. It begins around $x=200$ in the simulation, and ends roughly at $x=100$. In the experiment this 'tail' begins at $x=180$ and extends to $x=100$.

The comparison of these data with theoretical predictions, presented by Gajjar et al. [46], strongly suggests that the feature of the narrow large-particle 'tail' extending into the small-particlerich tail of the flow can be attributed to the effect of size-segregation asymmetry $[47,48,74]$. If large particles that are deposited on the bed move further upstream before they are re-entrained, they enter a region with many small particles. There they will segregate much slower because they are surrounded predominately by small particles. Consequently these large particles can be carried far back upstream before moving back downstream. On the other hand, small particles that enter the large-particle front segregate comparatively fast - as is characteristic of size-segregation asymmetry - thereby giving rise to the obtuse or rounded shape of the BSS wave on the side of the large-particle front. In Sec. 4.4 we show that in the experiment the mean flow velocity is reduced in the large-particle front, which could be given as an alternative explanation for the features that we attribute here to size-segregation asymmetry. However, the fact that the same features can be observed in the simulation, where the flow field does not change along the channel, makes this a more unlikely explanation. One more characteristic feature is the lower proportion of small particles at the free surface compared to the bed. For a detailed comparison between these data and theoretical predictions we refer the reader to the work of Gajjar et al. [46]. 


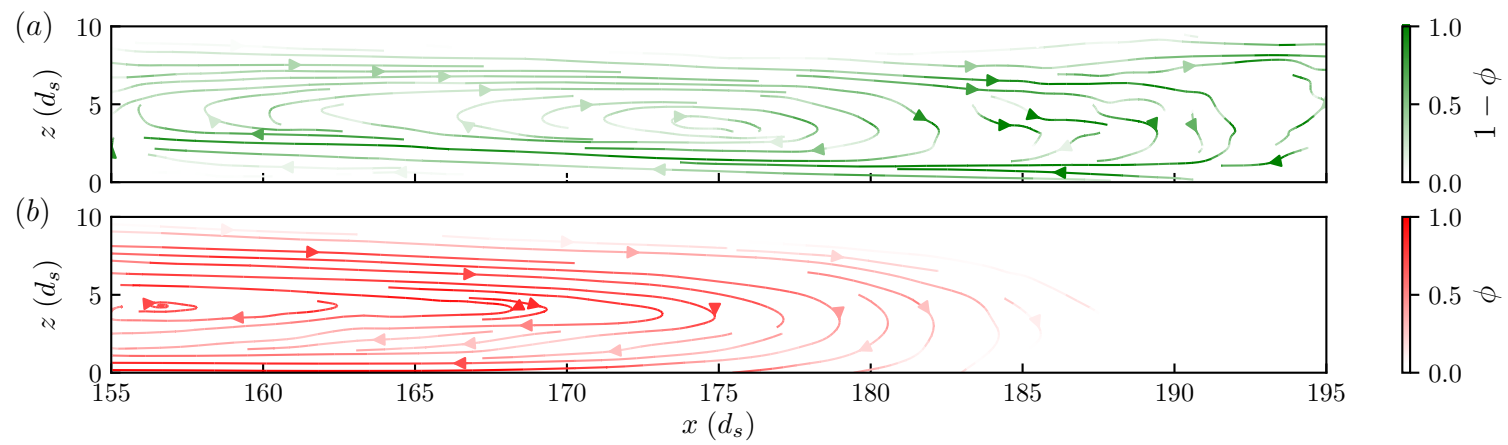

Fig. 6 Experimental data. Streamlines for large $(a)$ and small $(b)$ particles, at $\Phi=70 \%$. Faded arrows indicate a lower local volume fraction of that species.

We want to emphasise that the qualitative similarity between the experiment and simulation is impressive. No calibration was done, only the flow geometries are similar and the fact that both mixtures are bidisperse. This suggests that BSS waves are a very robust phenomenon. We suspect that the major differences between the structure of the BSS wave in the experiment and simulation can be attributed mainly to the effects of the shallower flow in the experiment and the difference in size-ratio.

\subsection{Intermezzo: The Perfect Wave}

Before discussing the data in more detail it is valuable to consider the ideal BSS wave in our geometry. Under ideal circumstances large particles and small particles segregate and recirculate before reaching the up- and downstream walls, respectively. In that case the BSS wave is isolated from the upper and lower boundaries and fits in the channel. This is the optimal situation to study a BSS wave because there is no artificial rising and sinking of large and small particles at the walls. We found that achieving the goal of isolating the BSS wave from the up and downstream walls is quite difficult, mainly because of the dramatic scaling of the length of the BSS wave with various parameters. Careful finetuning of the flow depth, particle sizes, size ratio, the global small-particle volume fraction $\Phi$, and channel inclination is necessary to obtain an isolated BSS wave. We will qualitatively discuss below the general effects of changing these parameters on the length of the BSS wave. We leave a more detailed study on the precise scalings for future work, but here want to qualitatively outline our findings in the preliminary testing.

In our preliminary testing we found that the formation of a large-particle front, with small particles never reaching the downstream wall, is quite robust. In contrast, obtaining a BSS wave where the large particles do not reach the upstream wall is more challenging. We found that, as a result of size-segregation asymmetry [47, 48], the large particles can travel very far upstream through the small-particle tail. Hence a pure small-particle tail is difficult to form. The upstream travel distance of large particles can be reduced by decreasing the flow depth, which is not preferable, or by increasing the segregation strength of large particles. The latter can be done by optimising the size ratio: A big size ratio facilitates an easier front formation because small particles segregate faster, however rising of isolated large particles in the tail is slower. A small size ratio, on the other hand, reduces segregation speeds for both species. The reason for this seeming contraction is that size-segregation in dense granular flows has an optimal strength at an intermediate size ratio of around $2.0[49,75]$. Ultimately, through trial and error in our preliminary tests we arrived at the size ratios used in the current study.

Another way to manipulate the distance between the centre of the BSS wave and the upstream wall is by varying the small-particle volume fraction $\Phi$. If $\Phi$ is increased, there are fewer large particles in the flow and the centre of the BSS wave will move downstream. However, if the 


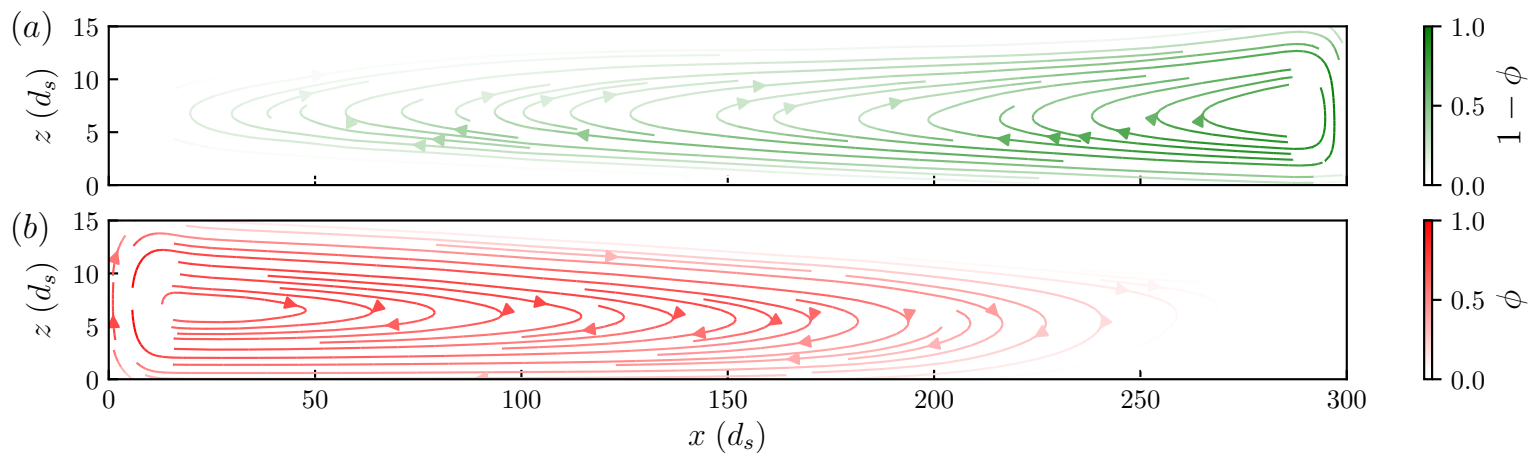

Fig. 7 Simulation data. Streamlines for large $(a)$ and small $(b)$ particles, at $\Phi=70 \%$. Faded arrows indicate a lower local volume fraction of that species. Because of the difference in flow and particle parameters between the experiment and simulation, only a qualitative comparison is possible between this figure and Fig. 6 .

amount of large particles is too low, no purelargeparticle front will form.

Adjusting the channel inclination, and thereby the flow velocity (since these are linked if we are to maintain uniform depth), has a complex effect on the upstream travel distance of large particles. A higher velocity results in a more kinetic flow, thus increasing the segregation speed, however, deposited large particles are also carried further upstream by the faster flow. Higher flow velocities also increases deposition of large particles on the bed near the front. This reduces the size of the large-particle front and increases the size of the BSS wave.

Our understanding of particle-size segregation is such that we can qualitatively explain the above described behaviours. Thornton and Gray [37] predicted that the size $W$ of a BSS wave scales as:

$W=\frac{1}{S_{r}}\left(1-\alpha_{s l i p}\right) h^{2}$,

where $h$ is the height of the BSS wave and in this case the flow depth, $\alpha_{\text {slip }}\left(0 \leq \alpha_{\text {slip }}<1\right)$ is the amount of basal slip, with $\alpha_{\text {slip }}=0$ corresponding to zero slip and $\alpha_{\text {slip }}=1$ to a plug flow. Formally we exclude the case of plug flow because no BSS wave will form in that case [37] and $W=0$. The parameter $S_{r}$ is the non-dimensional segregation number [44] defined as $S_{r}=L Q / h U$, where $L$ is a typical length of the entire avalanche, $Q$ is the magnitude of the segregation velocity, and $U$ is the magnitude of the downstream velocity. $S_{r}$ describes the ratio of a typical downstream transport time scale $L / U$, to a typical time scale for segregation $h / Q$. Note that a mod- ified version of Eq. (1), that takes into account size-segregation asymmetry, is derived in Gajjar et al. [46].

The size $W$ refers to the size of the lens region of the BSS wave (see Fig. 2). According to Eq. (1) the size of the lens is proportional to the shear rate through $1-\alpha_{\text {slip }}$, inversely proportional to the segregation number $S_{r}$ and proportional to the flow depth. In other words stronger segregation makes the BSS wave smaller, while higher shear and a deeper flow make it longer. Because $W \propto h^{3}$ it was necessary to use a low flow depth in the experiments and simulations that is perhaps far from realistic, being only a few large particle diameters near the front. However, this was required in order to create BSS waves that are isolated from the up- and downstream walls.

The experimental data is obtained from an isolated BSS wave, i.e., one for which the large particles do not reach the upstream wall and the small particles do not reach the downstream wall. This is also the case for the simulation data discussed so far and those discussed in the following two sections. In Section 4.6 and 4.7, however, we study the effect of varying $\Phi$ in the simulations. Only for some of the values of $\Phi$ the BSS wave fits in the channel. For other values no pure front or tail form, but nonetheless segregation occurs and a BSS wave forms. Even though these flows do not exhibit isolated BSS waves, the investigation yields some valuable results. Before looking into that we will first discuss in more detail the measured particle recirculation in a BSS wave. 

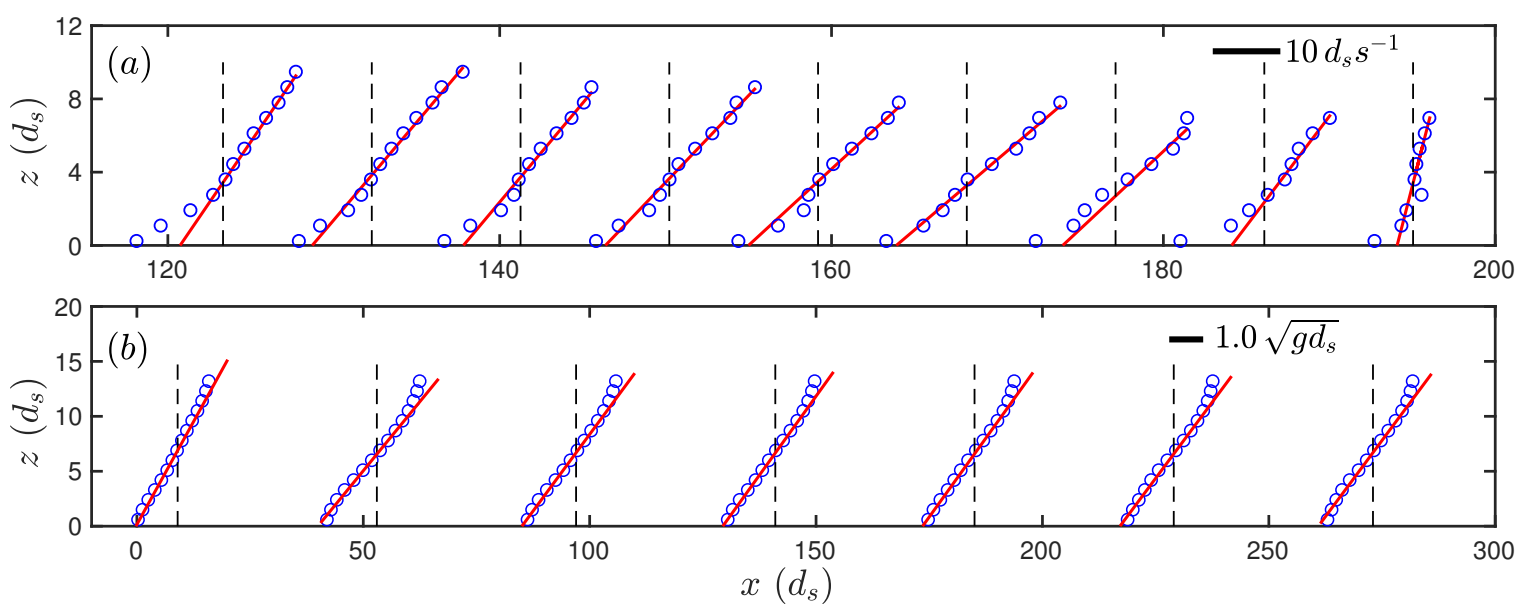

Fig. 8 Experimental and simulation data. The downslope velocity $u(z)$ as a function of the height $z$ at different $x$-positions for $(a)$ the experiment and $(b)$ the simulation, both at $\Phi=70 \%$. The dashed lines indicate the $x$-positions at which the data are measured, and it also corresponds to $u(z)=0$ for these data. The velocity scale is indicated by the thick black bars. Red dashed lines are fits of $f(z)=a z-b$, where $a$ and $b$ are constants. Note that the downstream wall in the experiment is at $x=208$, while it is at $x=300$ in the simulation. Because of the difference in flow and particle parameters between the experiment and simulation, only a qualitative comparison is possible here.

\subsection{Particle Recirculation}

The local small-particle volume fraction $\phi(x, z)$ discussed in Section 4.1 provides a detailed look at the structure of a BSS wave. However, it is not clear from these data how particles move and how recirculation takes place between the front and tail. Therefore we look at streamlines of the small and large particle phases in this section.

Fig. 6 shows streamlines, obtained from the coarse-grained partial velocity fields in the experiment, revealing the movement of the two phases. Sinking of small particles occurs predominantly in the region between $x=170$ and $x=190$. Large particles rise below $x=175$. Streamlines obtained from the simulations show qualitatively similar behaviour in Fig. 7. The region where small particles sink most strongly is just to the right of the region where large particle rising is strongest. One noteworthy difference between the simulation and experimental streamlines is that the experimental streamlines show some downward movement of large particles in the same region as where small particles move down, around $x=185$. This is not the case in the simulation. We attribute this difference to the fact that more basal slip occurs in the experiment (more on this in the next section) and the flow is more shal- low. The large-particle front in the experiment is much like a solid mass with a relatively lower shear-rate compared to other parts of the flow. As a consequence of this behaviour, some large particle circulation towards the bed occurs at the start of the large-particle front. Marks et al. [45] reported similar trajectories for a single large intruder in 2D granular avalanche in a moving-bed channel. In the next section we discuss the downslope velocity profiles for the BSS wave in the experiment and simulation.

\subsection{Downstream Velocity Profile}

Fig. 8 shows a comparison of the height-dependent downstream velocity $u(z)$ at different $x$-positions along the channel in the experiment and simulation. The vertical dashed lines indicate the $x$ positions where the profile is measured and also corresponds to $u(z)=0$ for the profile at that point. The bottom part of each $u(z)$ profile lies to the left of the dashed line, thus indicating a negative (upstream) velocity. Vice versa for the top part of each $u(z)$ profile, which has a positive (downstream) velocity.

The experimental data, in Fig. 8(a), shows a linear scaling with depth, only deviating from linearity close to the bed, where the shear rate 

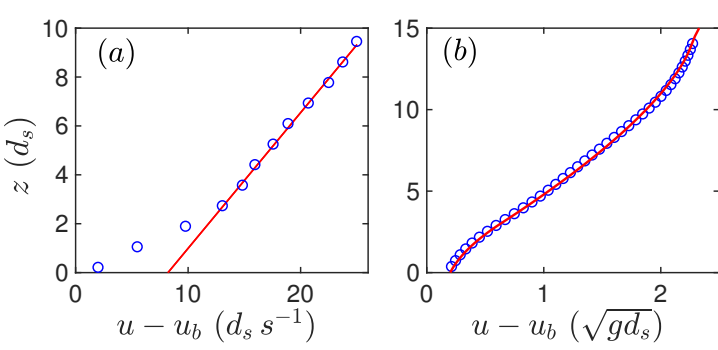

Fig. 9 Experimental and simulation data. Horizontal velocity $u(z)$ minus $u_{b}$ as a function of height $z$, at $x=20$ and $\Phi=70 \%$, for $(a)$ the experiment, and $(b)$ the simulation. Red dashed line in $(a)$ is a fit to the top of the flow with $f(z)=a z-b$, where $a$ and $b$ are constants, while in (b) the fit is of Eq. (55b) in [62].

$\dot{\gamma}=\partial u / \partial z$ increases. For a shallow flow such as this, a linear velocity profile is to be expected [62, $76,77]$, in contrast to a Bagnold profile, where $u(z)$ scales with $(h-z)^{3 / 2}[78]$. At higher $x$, the velocity profile steepens, which is likely the result of a slight reduction in flow depth. Above $x=180$ the mean velocity suddenly decreases. This can be attributed to a basal slip between the mixture and the bed. The roughness of the bed in the experiment is relatively small compared to the size of the large particles. Hence, the small-particle tail experiences a different amount of basal friction compared to the large-particle front. We do not view this behaviour as an artifact, since large boulders are known to be pushed en masse in front of debris flows $[13,79,80]$. In fact as we will see shortly, the tail also experiences a basal slip, albeit less. Interestingly, we find that the overall effect of the decreased shear rate in the large-particle front is to promote its formation: a slower-moving front makes it more likely that a small particle has segregated to the bed before it can reach the downstream wall.

The simulation data, in Fig. 8(b), does not exhibit the same decrease in mean velocity in the downstream end of the flow. We attribute this to the fact that we were able to more carefully choose a roughness of the bed such that the difference in traction between a purely large and purely small mixture was less compared to that in the experiments. The velocity in the simulations is reasonably well fitted with a linear function, however, near the free-surface and close to the bed the velocity is slightly reduced and deviates from the linear trend of the fit. This trend is qualitatively different from the one observed in the experimental data and is reminiscent of the observations of Weinhart et al. [62], who found three different regimes in the velocity profile for thick flows; a linear part near the free-surface, a quadratic part near the bed, and a Bagnold scaling in between. For shallow flows, the linear and quadratic regimes would connect to give a nearly linear profile along the entire depth. In Fig. 9 we plot a single experimental and simulation velocity profile measured at $x=20$. These profiles are shifted with $u_{b}$ in order to demonstrate the finite basal slip. To compare the basal slip in the experiment and simulation, we define a slip velocity $u_{\text {slip }}=u(z=0)-u_{b}$. The non-dimensional slip velocity $u_{s l i p} / u_{b}$ is approximately 0.16 in the simulation and 0.14 in the experiment. We will further discuss the basal slip in Sec. 4.6.

Fig. $9(b)$ demonstrates that the functional dependence for $u(z)$ proposed by Weinhart et al. [62] fits the velocity profile in the simulation quite well. The fact that the experiment exhibits a purely linear velocity profile and the simulation does not, can reasonably be attributed to the difference in flow height in terms of particle diameters [62].

\subsection{Flow Depth}

Despite our aim to maintain a uniform flow depth along the length of the channel a variation of the depth with $x$-position does occur in the simulations for mixtures with $\Phi$ between $10 \%$ and $90 \%$. Fig. 10 shows that for $\Phi=0 \%$ and $100 \%$ the flow depth is practically constant (to within half a small-particle diameter) but that for intermediate values of $\Phi$ the depth increases slightly at the downstream and upstream walls and decreases in the centre of the flow by up to a maximum of around $4 d_{s}$ for $\Phi=30 \%$. Readers should be aware that the slope of the free-surface is greatly exaggerated in the figure as a result of the aspect ratio of the plot. The decrease in the flow depth is strongest for mixtures with $\Phi$ between $30 \%$ and $50 \%$, suggesting that it is related to the full formation of the BSS wave. As a side note, we also observe that between $\Phi=10 \%$ and $50 \%$ the position of the minimum in flow depth $h$ shifts toward higher values of $x$ before it disappears. 


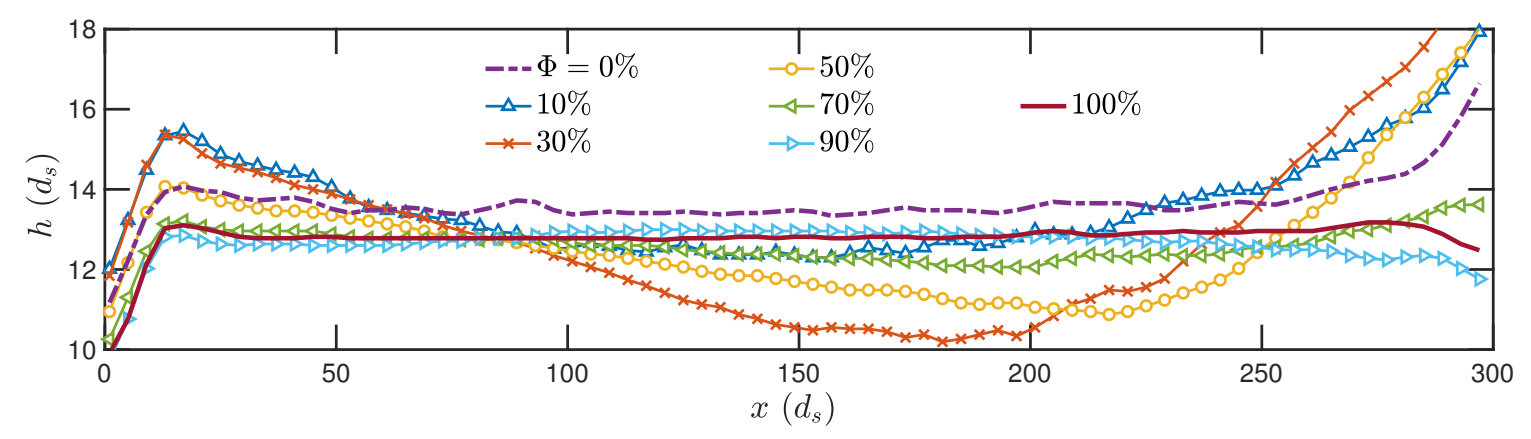

Fig. 10 Simulation Data. Flow depth $h$ as a function of $x$ for varying $\Phi$.

The reason for this effect is unclear at this point. Judging from coarse-grained density fields (data not included) the observed flow depth decrease can not simply be attributed to a higher packing fraction resulting from the local mixing of large and small particles; for that the reduction in flow depth is too big. On the contrary, we observe that the packing is slightly less dense in the region where the dip in flow height occurs.

Another explanation for the non-uniform flow depth at intermediate $\Phi$ could be that this behaviour is inherent to the flow geometry, or resulting from our method for tuning the belt speed. This method (described in more detail in Sec. 3.3) is to measure the distance of the centre-of-mass of the flowing mixture to the centre of the channel and update the belt speed proportionally. A steady-state configuration with the centre-of-mass at $x=150$ is possible if the front and tail of the flow are slightly deeper compared to the centre. Currently we cannot explain why a flow with a more fully developed BSS wave prefers this state over a more uniformly deep one. Possibly it is linked to a change in local bulk friction, as Pouliquen [81] and Gray and Ancey [38] showed a relation exists between the free-surface slope $d h / d x$ and the local bulk friction. Saingier et al. [82] generalised this relation for a non-plug flow.

In the next section we further investigate the basal slip.

\subsection{Basal Slip}

We plot $u_{\text {slip }}(x)$ in Fig. 11(a) for three different simulations with varying global small-particle volume fraction $\Phi$ and belt speed $u_{b}$. We observe that the slip is non-zero along the entire length of the channel for all three flows. The slip appears to depend on both $x$ and $\Phi$, but no clear trend is immediately evident. Weinhart et al. [62] reported a scaling of basal slip with the mean velocity in their chute flow simulations. Thus we normalise the data with $u_{b}$ as well as with the inverse of the flow depth $h(x)$, since $h$ is not perfectly uniform along the channel. Fig. 11(b) shows that after this normalisation the data collapse onto two plateaus. For $\Phi=0$, when the entire flow is composed of large particles, the basal slip is constant over the entire length of the channel, and at the upper plateau value. When $\Phi$ is increased, the slip is first reduced in the upstream part of the flow (low $x$ ), while the downstream part is unaffected and remains at the level of the upper plateau value. As $\Phi$ is further increased the slip gradually reaches the lower plateau value in the upstream part of the flow, while the extend of the upper plateau is pushed further downstream, to higher $x$ positions. At approximately $\Phi=70 \%$ the upper plateau has disappeared entirely, with only a slight gradual increase in slip above $x=100$.

It is reasonable to associate the two plateaus of the basal slip and the dependency on $\Phi$ with the local presence of the two particle species, where the large-particle front experiences more slip compared to the small-particle tail. In Fig. 12, we plot $u_{\text {slip }}(x) h(x)$ for $\Phi=40 \%$ together with $\phi(x, 0)$ and $1-\phi(x, 0)$ in the same flow. Indeed, the increase of $u_{\text {slip }}(x)$ begins where $\phi(x, 0) \approx 0.9$ and saturates where $\phi(x, 0)=0$ within $\pm 10 d_{s}$. Jing et al. [83] reported a similar correlation between the basal slip and the degree of local segregation in a chute flow. They found that when small particles sink to the bed in a region previously dominated by large particles the basal slip is re- 


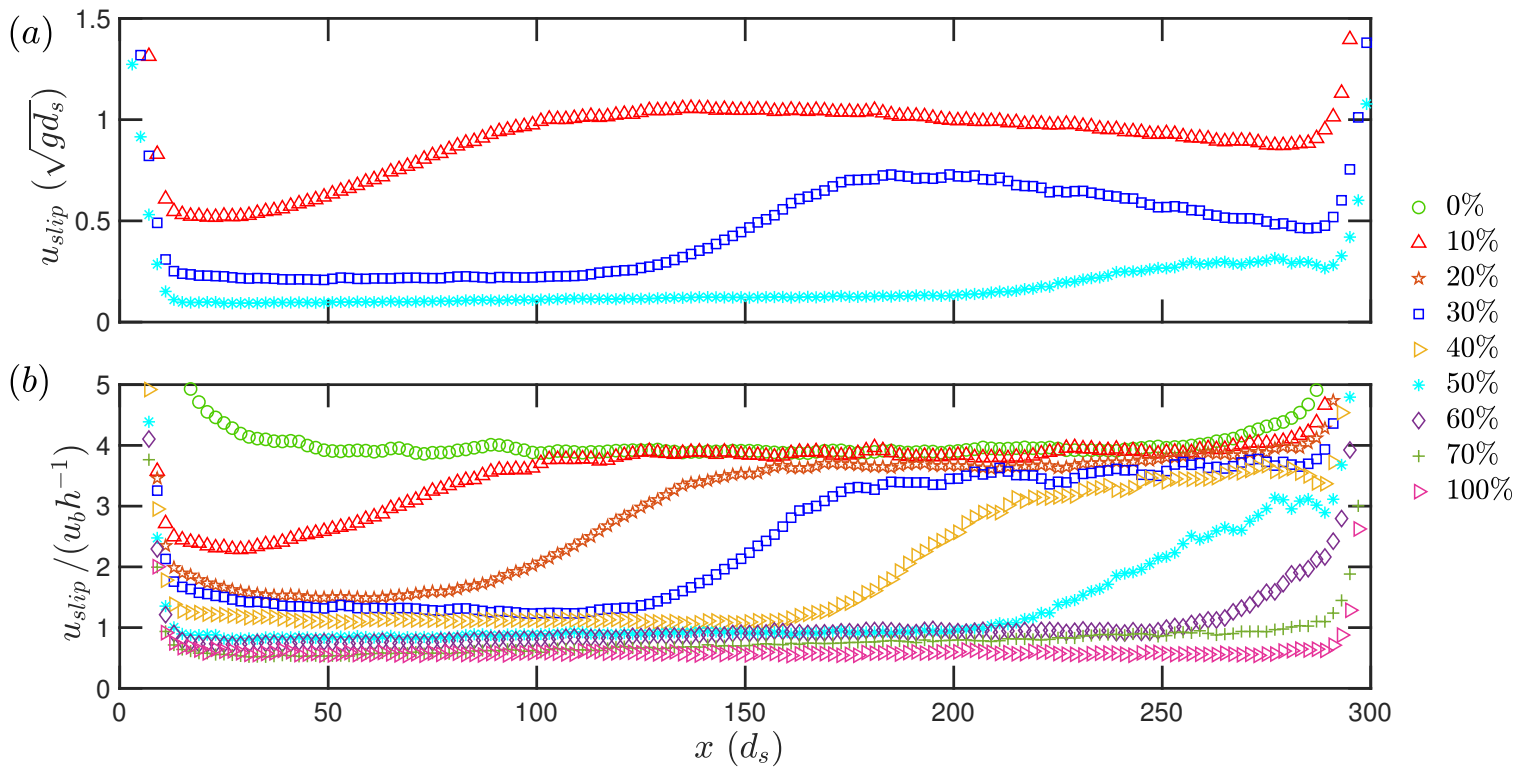

Fig. 11 Simulation data. (a) Slip velocity $u_{s l i p}=u(z=0)-u_{b}$ in the simulations for three different global small-particle volume fractions $\Phi$. (b) Slip velocity in the simulations normalised by $u_{b} h^{-1}$, for mixtures of varying $\Phi$ and $u_{b}$. The values of $u_{b}$ for each $\Phi$ can be found in Fig. 13(a).

duced. Interestingly, Jing et al. [83] also found that the rate of segregation is reduced following an increase in basal slip as this in turn reduces the shear-rate. These findings suggest that the large-particle-rich front is less resistive to flow than the tail, i.e., it flows easier because it slips more. Interestingly, in the process of finger formation $[12,14,15]$ the flow-front experiences an increased traction with the bed, due to the presence of the rougher more angular large grains, which is contrary to what seems to happen here. However, during finger formation the flow also increases in height in order to subsequently reduce the increased resistance the flow feels from the bed. Since in our experiments and simulations the flow height is controlled another way for the flow to reduce the flow resistance is to slip, which is precisely what happens.

A crucial observation in the discussed data is that the fraction of the bed that experiences the reduced basal slip because of the presence of small particles, is always bigger than the fraction of small particles in the mixture. This seems to be a direct result of the structure of the BSS wave where small particles are predominantly positioned near the bed, as demonstrated by the data of $\phi(x, 0)$ in Fig. 12 and Fig. 5(d), as well as by theoretical predictions $[37,46]$.

\subsection{Mobility Feedback}

In the context of particle-size segregation, the effect of mobility feedback refers to the feedback that exists between the bulk flow and particlesize segregation [14, 24, 39, 42]: The rate of sizesegregation is affected by the local flow velocity, while the flow velocity is affected by the local volume fraction of small and large particles. In

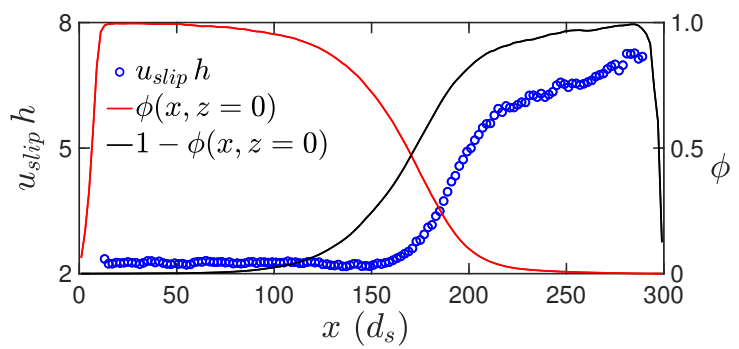

Fig. 12 Simulation data. Slip velocity $u_{\text {slip }}=u(z=$ $0)-u_{b}$ normalised by the inverse of the flow depth $h(x)$, for $\Phi=40 \%$ plotted together with $\phi(x, 0)$ and $\phi_{l}(x, 0)=1-\phi(x, 0)$. The decrease of $\phi$ and $1-\phi$ at $x=0$ and $x=300$ is a boundary effect due to the walls. 

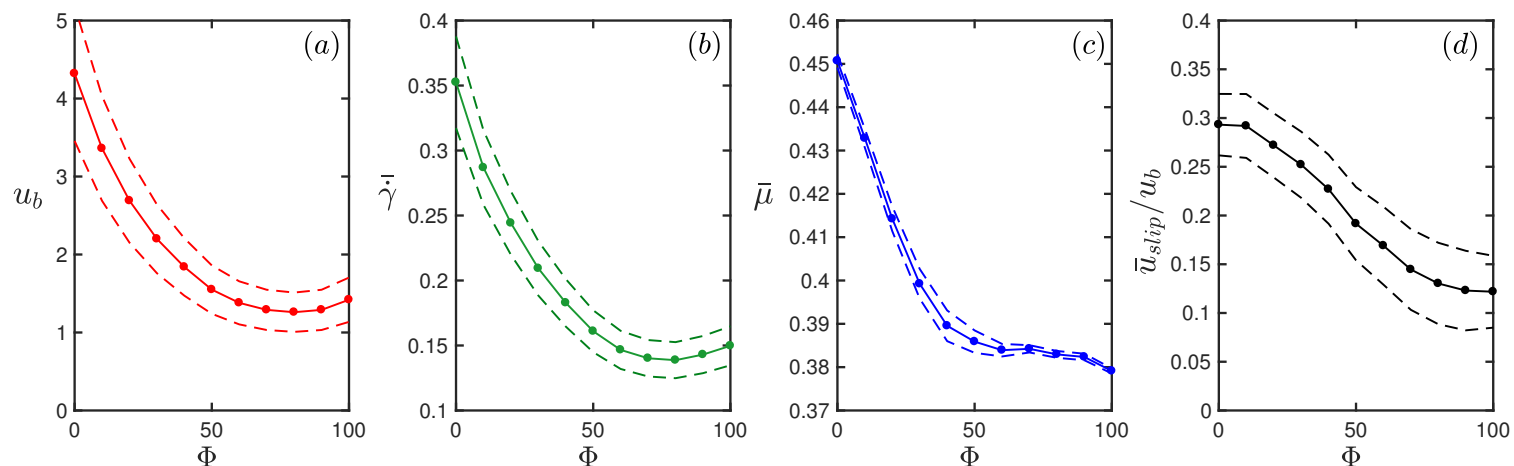

Fig. 13 Simulation data. (a) The belt speed $u_{b}$ as a function of $\Phi$. The belt speed is continuously adapted in order to maintain a uniform depth and therefore fluctuates in time. (b) The shear-rate $\overline{\dot{\gamma}}$, averaged along the length of the channel, plotted as a function of $\Phi$. (c) The depth averaged bulk friction $\bar{\mu}$, averaged along the length of the channel, plotted as a function of $\Phi$. $(d)$ The average slip velocity $\bar{u}_{\text {slip }}$, averaged along the length of the channel and normalised by $u_{b}$, plotted as a function of $\Phi$. The dashed lines correspond to the standard error of the mean.

turn, the local volume fraction naturally evolves through size-segregation, thus creating a feedback loop.

Here we will discuss observations that indicate feedback between the flow velocity of the mixture, the structure of the BSS wave and the concentration of small particles in the flow. Central to this discussion is the relation between the global small-particle volume fraction $\Phi$ and the belt speed $u_{b}$ required to maintain a uniform depth. The dependence of $u_{b}$ on $\Phi$ for the simulations is plotted in Fig. 13 $(a)$. We see that $u_{b}$ is maximum at $\Phi=0$, when the flow is entirely composed of large particles. Upon increasing $\Phi, u_{b}$ is decreased. The decrease in $u_{b}$ saturates above $\Phi=60 \%$ where $u_{b}$ remains more or less constant and slightly increases at $\Phi=100 \%$. If we were to shift our reference frame by $u_{b}$, such that the downslope velocity at the bed is zero instead of negative, and all particles have a positive downstream velocity, the depth-averaged flow velocity $\bar{u}=\left\langle u+u_{b}\right\rangle_{h}$ will be equal to the $u_{b}$. Hence, changing the belt speed is equivalent to changing the mean flow velocity in the system. In Fig. 13(b) we plot the depth-averaged shear-rate $\overline{\dot{\gamma}}$ as a function $\Phi$, showing a similar dependence on $\Phi$ as $u_{b}$.

To understand the decrease of the belt speed and mean flow velocity with $\Phi$ we consider how adding small particles to the flow affects the depthaveraged bulk friction $\bar{\mu}$. Rognon et al. [28] and Staron and Phillips [84] showed that bidisperse granular flows with a higher concentration of small particles exhibit a lower bulk friction. The data of $\bar{\mu}$, averaged along the length of the channel, is plotted in Fig. 13(c) and demonstrates that indeed in our system the bulk friction is also lowered by the presence of small particles. When $\Phi$ increases and $\bar{\mu}$ decreases, the transfer of momentum from the bed to the flow is more efficient, hence, more material is dragged upslope and accumulates at the upstream wall. Subsequently, the belt speed and mean flow velocity decrease to maintain a uniform depth. As discussed in the previous section the increase of $\Phi$ is accompanied by a decrease in slip velocity $\bar{u}_{\text {slip }}$ in the tail of the flow, which shows up as a decrease in the average slip velocity (normalised by $u_{b}$ ) in Fig. 13(d). This effect also contributes to the increase of transfer of momentum from the bed to the flow. These data demonstrate a tight interplay between the bulk composition and the bulk mobility.

The belt speed, shear-rate, bulk friction and average slip velocity all exhibit a non-monotonic decrease and subsequent saturation above $\Phi=$ $70 \%$. This effect might be linked to the compositional structure of the BSS wave. Namely, above $\Phi=70 \%$ the bed is nearly completely saturated with small particles, as discussedin the previous section. Thus, any further addition of small particles will not decrease the basal slip any more. Since the strongest contribution to the bulk friction comes from layers near the bed, further addition of small particles will not reduce $\bar{\mu}$. The 
observed saturation suggests that the effect of $\Phi$ on the bulk mobility is strongly related to the location of the small particles in the flow and thus to the structure of the BSS wave.

\subsection{Bulk Friction and Position}

Before concluding we want to discuss another feature for which we do not have an explanation at this point, but is nonetheless very interesting. The data in Fig. 13(c) of the depth-averaged bulk friction, averaged along the length of the channel, hides the fact that $\bar{\mu}$ varies with $x$. This variation is plotted in Fig. 14(a) for different $\Phi$ for the simulations. At $\Phi=0 \%$ and $100 \%$ the friction is constant with $x$, as is to be expected, since the flow is monodisperse. However, at intermediate values of $\Phi$ the friction varies with $x$. For $x<150$ we observe that increasing $\Phi$ decreases $\bar{\mu}$ and that a saturation occurs at around $\Phi=70 \%$. This is the same trend as observed in the $x$-averaged $\bar{\mu}$ in Fig. $13(c)$. However, above $x \approx 150$ the friction decreases much stronger with $\Phi$ and becomes even smaller than for $\Phi=100 \%$. The minimum is at around $\Phi=50 \%$. Subsequently, the friction in this frontal region increases again, until at $\Phi=70 \%$ it saturates to the same value as in the upstream region $(x<150)$.

This behaviour is remarkable because the friction in the large-particle front decreases even though locally the concentration does not actually change; the small particles that are added to the flow accumulate first and foremost in the tail, as can be seen in Fig. 14(b), where the depth-averaged local small-particle volume fraction $\bar{\phi}$ is plotted as a function of $x$. The increase of $\bar{\mu}$ in the front region of the flow, when $\Phi>50 \%$, does coincide with small particles reaching that part of the flow. The question why the friction in the front is decreased for $\Phi<50 \%$ while the local composition does not change, we cannot answer at this point. The BSS wave seems to have a non-local effect on the friction that is not captured by the current coupled models of segregation.

\section{Discussion}

Goujon et al. [85] showed for monodisperse flows that bed roughness, defined by them as the ratio
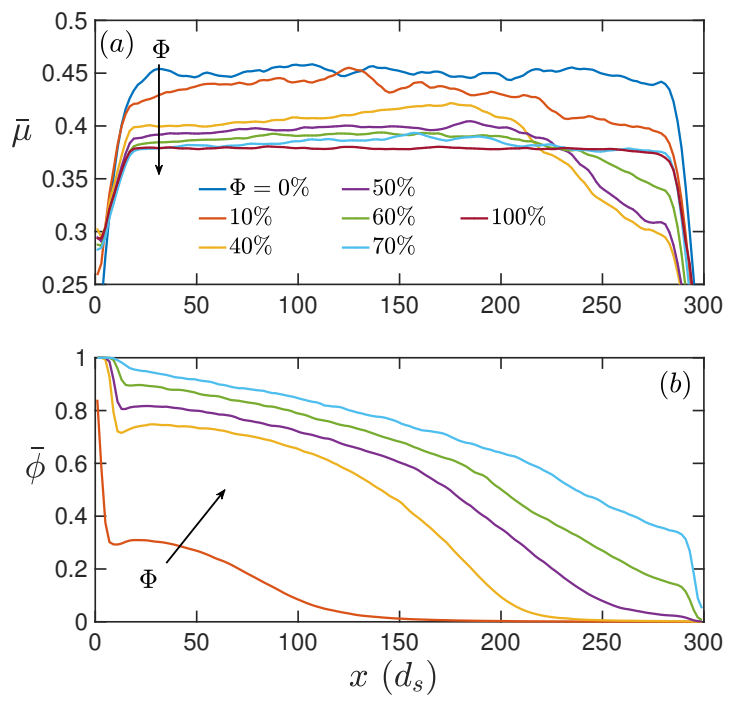

Fig. 14 Simulation data. (a) Depth-averaged bulk friction $\bar{\mu}$ as a function of $x$ for varying global smallparticle volume fractions $\Phi$. (b) Depth-averaged local small-particle volume fraction $\bar{\phi}$ as a function of $x$ for varying global small-particle volume fractions $\Phi$. The legend in $(a)$ is also for the data in $(b)$.

between the size of flowing particles $d_{p}$ and the size of bed particles $d_{b}$, is the main parameter determining basal friction. They found a maximum friction between the bed and the flow around $d_{b} / d_{p}=2$. If $d_{p} \gg d_{b}$ the basal friction decreases, while for $d_{p} \ll d_{b}$ the friction is also decreased, because holes in the bed are filled with small particles, thereby reducing the roughness. For the data presented here $d_{b} / d_{s}=1.7$ and $d_{b} / d_{l} \approx 0.71$. This would explain why the large particles experience less friction than the small particles: they are further away from the optimum diameter ratio $d_{b} / d_{p}=2$. Weinhart et al. [62] also reported weak to stronger slip velocities for $d_{b} / d_{p}<0.67$ in their DPM simulations with monodisperse flows. For bidisperse flows, Goujon et al. [24] reported a modification of the basal friction depending on the size of the beads that are in contact with the bed. This influenced the spreading and fingering of the flow.

More recently, Jing et al. [86] introduced a characterisation of bed roughness considering both the size and spatial distribution of bed particles. They proposed a roughness parameter $R_{a}$ that combines both factors in two- and threedimensional configurations. When $R_{a}$ increases 
in three-dimensional mono-disperse flows a transition occurs from $\operatorname{slip}\left(R_{a}<0.51\right)$ to non-slip $\left(R_{a}>0.62\right)$ conditions. In a two-dimensional flow, the critical values are 0.7 and 0.75 , respectively. Jing et al. [83] validated such a transition in bidisperse flows, where $R_{a}$ becomes a function of time following the progress of segregation. Similar to mono-disperse flows, basal slip arises at low values of $R_{a}$, which might lead to a delayed development of segregation; when $R_{a}$ increases beyond 0.62 , no slip can be observed. Following the characterization in [86], we estimate the roughness $R_{a}$ in our configurations to be $0.5-0.8$ in the simulations and $0.4-0.64$ in the experiments, depending on the local flow composition. As such, a slip would occur when more large particles appear near the base, while it becomes less likely with more small particles.

The BSS wave is a transition region between the small-particle tail and large-particle front of an avalanche. The findings reported here demonstrate that the compositional structure of this transition region determines how the bulk friction and traction vary along the avalanche length from the front to the tail. This insight is important for the theoretical modeling of mobility feedback. In the theoretical framework of Gray and Kokelaar $[39,40]$ a coupling or feedback between the bulk velocity field and size-segregation can be accomplished by making (i) the basal friction, (ii) the basal deposition rate of particles, (iii) the velocity shape profile or (iv) the granular viscosity, dependent on the local composition of the flow. Our findings show that only the basal friction appears to be a strong function of local composition; suggesting this is the only dependence on local small-particle volume fraction $\phi$ that needs to be included in a coupled framework. However, the dependence on $\phi$ is complex and does depend on the whole $\phi(z)$ profile not just the $\phi$ at the base or the mean value of $\phi$. This raises interesting questions as to how, or even, if it is possible to include this dependence in a depth-averaged framework like the one presented by Gray and Kokelaar [39, 40].

In the framework of Gray and Kokelaar [39, 40] and similar depth-integrated particle transport models $[15,36]$, the vertical distribution of $\phi$ is assumed to be rapidly established. While this assumption may be justified in many regions of a flow, it causes a BSS wave to be resolved in these models as a shock, which implies a discontinuous change in bulk friction at the centre of the BSS wave [39]. Our study indicates that the bulk friction instead varies over a lengthscale of the same order as the width of the BSS wave, or to the width of the transition between $\phi=0$ and $\phi=1$ at the base of the flow. This lengthscale of the BSS wave may play an important role in coupled flow and segregation models, since simple models of this sort (that approximate a BSS wave as a shock) predict granular fingering and levee formation at arbitrarily small scales [14]. In order to derive more accurate and predictive coupled flow and segregation models the lengthscales introduced by the BSS wave, as well as those arising from viscosity [15], may have to be incorporated.

Another point of discussion is that of the comparison between the flow in the moving-bed channel and the flow on a normal inclined plane or in a channel. We find that the velocity profile in the moving-bed channel is very similar to that of a normal inclined plane flow. For thin flows a linear profile is expected, and the data we report can be fitted well with the form found by Weinhart et al. [62] for chute flows. We verified that when simulating a granular mixture flowing in a box with periodic walls in both the cross and downstream directions and a moving bed the velocity profile is simply shifted with respect to the flow on a stationary bed. Similarly, a simple shift of velocity profile caused by basal slip is found in normal chute flows [83].

\section{Conclusions and Future Prospects}

The data presented here provides the first direct evidence for the existence of the theoretically predicted breaking size-segregation (BSS) wave in experiments and simulations. We find that its formation and structure is robust, which, in combination with previously reported indirect evidence [21], is a strong argument for the existence of BSS waves in naturally occurring geophysical flows. The mobility feedback effects observed here underline the importance of studying BSS waves in the context of geophysical flows. Since the practical challenge of visualising BSS waves in naturally occurring flows has not been 
resolved, smaller experiments and simulations like the ones performed here, will likely continue to play a critical role.

This work demonstrates that the moving-bed channel geometry is a powerful tool to study the effects that particle-size segregation has on the compositional structure and flow behaviour of polydisperse gravity-driven dense granular flows. Our findings indicate that the flow in a moving-bed channel is representative of a normal granular avalanche, however, a more quantitative comparison is needed to establish this irrefutably.

In our preliminary tests we found a strong increase of the length of BSS waves with a small increase in flow depth, suggesting a quadratic or cubic scaling. This is supported by theoretical predications of a super linear scaling of the length of BSS waves with their flow depth [37, 46]. Such a scaling results in BSS waves that are very long compared to the avalanche depth, which makes it difficult to contain one in a small-scale lab experiment. A quantitative verification of the scaling of the length of BSS waves with different parameters could be addressed in future studies. The asymmetric shape we observe for BSS waves can be explained by size-segregation asymmetry $[46,48]$ underlining the importance of this fundamental behaviour in the context of polydisperse granular avalanches.

The presence of a BSS wave introduces a complex interaction between segregation and the mobility of the bulk flow; which evolves over the entire length of the BSS wave. This length-scale may have to be incorporated in coupled flow and size-segregation (mobility feedback) models, in order to improve their predictive power. The inclusion of this length-scale is a challenge for the current depth-averaged models as the feedback on the bulk flow is a function of the full depthdependent segregation profile. The solution may be to extend the multilayer approach of Edwards and Vriend [36] to describe segregation profiles in which $d \phi / d z>0$ (small particles on top of large ones), as seen in BSS waves.

We believe it is important for future work to address the validity of our approach to choose the inclination angle and belt speed such that the flow depth along the length of the channel is uniform. This assumption, although ideal, is not the most realistic as naturally occurring granu- lar avalanches typically have a shallow tail and a more bulbous head [80, 87, 88]. Flows in longer moving-bed channels (experimental or simulated) could be investigated in an attempt to isolate the entire flowing mixture from the two end-walls and study the entirety of an avalanche.

The scale of the flows studied here also deserves some additional remarks. The complex scaling of the length of BSS waves with their flow depth, the segregation rate and other flow parameters, forced us to use a large particle-size ratio and a shallow flow depth, whereas naturally occurring granular avalanches typically have a bigger polydispersity and are much deeper in terms of particle diameters. We also did not incorporate an interstitial fluid that flowed with the avalanche, i.e., the interstitial fluid in the experiment is relatively stationary, therefore the effect of pore fluid pressure in natural debris flows is under-represented, whereas the effects of grain inertia are over-represented. We believe that future work could address this issue.

Ultimately, our findings show that BSS waves are a physically realisable phenomenon that researchers of polydisperse granular avalanches, debris flows and pyroclastic flows should take into consideration in their studies. The verification of the existence of BSS waves opens up the way for further detailed study, which will be invaluable for the development of models that capture the mobility feedback between particle-size segregation and bulk flow behaviour of avalanches.

The DPM code for running moving-bed channel simulations is available at MercuryDPM.org. The authors declare no conflict of interest.

Acknowledgements This study was funded by the Dutch STW VIDI project No. 13472, the Swiss SNF Grant No. 200021-149441, and by NERC grants NE/E003206/1 and NE/K003011/1 as well as EPSRC grants $\mathrm{EP} / \mathrm{I019189/1,} \mathrm{EP/K00428X/1} \mathrm{and} \mathrm{EP/M022447/1.}$ J.M.N.T.G. is a Royal Society Wolfson Research Merit Award holder (WM150058) and an EPSRC Established Career Fellow (EP/M022447/1). The authors are grateful to B. de Graffenried for technical assistance, to M. Teuscher for designing and building the experimental setup, and to J.-L. Pfister for assistance with the experiments and analysis. The authors also thank H. Capart for providing the Voronoï tracking code and the referees for helping to improve this paper. 


\section{References}

1. R. P. Sharp and L. H. Nobles. Mudflow of 1941 at wrightwood, southern california. Geological Society of America Bulletin, 64(5):547-560, 1953.

2. R. A. Bagnold. Deposition in the process of hydraulic transport. Sedimentology, 10(1):45-56, 1968.

3. T. Takahashi. Debris flow on prismatic open channel. Journal of the Hydraulics Division, ASCE, 106(3):381-396, 1980.

4. T. Takahashi. Debris flow. Annual Review of Fluid Mechanics, 13:57-77, 1981.

5. A. M. Johnson. Physical Processes in Geology. Freeman, Cooper \& Company, 1970.

6. A. M. Johnson. Debris flows. In Slope Instability. Wiley, 1984.

7. J. E. Costa and G. Williams. Debris flow dynamics. Technical Report 84-606, (videotape) U.S. Geological Survey, 1984.

8. R. M. Iverson, M. Logan, R. G. LaHusen, and M. Berti. The perfect debris flow? aggregated results from 28 large-scale experiments. Journal of Geophysical Research, 115:F03005, JUL 102010.

9. R. M. Iverson. Debris flows: behaviour and hazard assessment. Geology Today, 30(1):15-20, 2014.

10. B. Turnbull, E. T. Bowman, and J. N. McElwaine. Debris flows: Experiments and modelling. Comptes Rendus Physique, 16(1):86-96, 2015.

11. T. C. Pierson. Flow behavior of channelized debris flows, Mount St. Helens, Washington, pages 269 296. Allen \& Unwin, 1986.

12. O. Pouliquen, J. Delour, and S. B. Savage. Fingering in granular flows. Nature, 386:816-817, 1997.

13. O. Pouliquen and J. W. Vallance. Segregation induced instabilities of granular fronts. Chaos, 9(3): 621-630, 1999.

14. M. J. Woodhouse, A. R. Thornton, C. G. Johnson, B. P. Kokelaar, and J.M.N.T. Gray. Segregationinduced fingering instabilities in granular freesurface flows. J. Fluid Mech., 709:543-580, 2012.

15. J. L. Baker, C. G. Johnson, and J.M.N.T. Gray. Segregation-induced finger formation in granular free-surface flows. J. Fluid Mech., 809:168-212, 2016.

16. L. Wilson and J. W. Head. Morphology and rheology of pyroclastic flows and their deposits, and guidelines for future observations. U.S. Geological Survey Professional Paper, (1250):513-524, 1980.

17. J. J. Major and R. M. Iverson. Debris-flow deposition: Effects of pore-fluid pressure and friction concentrated at flow margins. Geological Society of America Bulletin, 111(10):1424-1434, 1999.

18. E. S. Calder, R. S. J. Sparks, and M. C. Gardeweg. Erosion, transport and segregation of pumice and lithic clasts in pyroclastic flows inferred from ignimbrite at lascar volcano, chile. J. Volcanol. Geotherm. Res., 104(1):201-235, 2000.

19. G. Félix and N. Thomas. Relation between dry granular flow regimes and morphology of deposits: formation of levées in pyroclastic deposits. Earth and Planetary Science Letters, 221(1):197-213,
2004.

20. P. Bartelt, J. Glover, T. Feistl, Y. Bühler, and O. Buser. Formation of levees and en-echelon shear planes during snow avalanche run-out. $J$. Glaciology, 58(211):980-992, 2012.

21. C. G. Johnson, B. P. Kokelaar, R. M. Iverson, M. Logan, R. G. LaHusen, and J.M.N.T. Gray. Grain-size segregation and levee formation in geophysical mass flows. Journal of Geophysical Research, 117(F1):013301, 2012.

22. N. Thomas. Reverse and intermediate segregation of large beads in dry granular media. Physical Review E, 62(1):961-974, 2000.

23. B. Zanuttigh and A. Di Paolo. Experimental analysis of the segregation of dry avalanches and implications for debris flows. J. Hydraul. Res., 44 (6):796-806, 2006.

24. C. Goujon, B. Dalloz-Dubrujeaud, and N. Thomas. Bidisperse granular avalanches on inclined planes: A rich variety of behaviors. The European Physical Journal E: Soft Matter and Biological Physics, 23:199-215, 2007.

25. G.V. Middleton. Experimental studies related to problem of flysch sedimentation. In G.V. Middleton and A.H. Bouma, editors, Flysch Sedimentology in North America (Lajoie, J., ed.) Business and Economics Science Ltd., pages 253-272, 1970.

26. J. M. Ottino and D. V. Khakhar. Mixing and segregation of granular materials. Annual Review of Fluid Mechanics, 32(1):55-91, 2000.

27. S. B. Savage and C. K. K. Lun. Particle size segregation in inclined chute flow of dry cohesionless granular solids. J. Fluid Mech., 189:311-335, 1988.

28. P. G. Rognon, J.-N. Roux, M. Naaim, and F. Chevoir. Dense flows of bidisperse assemblies of disks down an inclined plane. Physics of Fluids, 19(5):058101, 2007.

29. L. B. H. May, L. A. Golick, K. C. Phillips, Michael Shearer, and Karen E. Daniels. Shear-driven size segregation of granular materials: Modeling and experiment. Physical Review E, 81:051301, May 2010.

30. S. Wiederseiner, N. Andreini, G. Epely-Chauvin, G. Moser, M. Monnereau, J.M.N.T. Gray, and C. Ancey. Experimental investigation into segregating granular flows down chutes. Physics of Fluids A, 23(1):013301, 2011.

31. B. Marks, P. Rognon, and I. Einav. Grainsize dynamics of polydisperse granular segregation down inclined planes. J. Fluid Mech., 690:499-511, 2012.

32. Y. Fan, Y. Boukerkour, T. Blanc, P. B. Umbanhowar, J. M. Ottino, and R. M. Lueptow. Stratification, segregation, and mixing of granular materials in quasi-two-dimensional bounded heaps. Physical Review E, 86(5):051305, 2012.

33. L. Staron and J.C. Phillips. Segregation time-scale in bi-disperse granular flows. Physics of Fluids, 26 (3):033302, 2014

34. J.M.N.T. Gray, P. Gajjar, and P. Kokelaar. Particle-size segregation in dense granular avalanches. Comptes Rendus Physique, 16(1):73- 
85, 2015.

35. D. R. Tunuguntla, T. Weinhart, and A. R. Thornton. Comparing and contrasting size-based particle segregation models. Comp. Part. Mech., pages 1-19, 2016.

36. A. N. Edwards and N. M. Vriend. Size segregation in a granular bore. Phys. Rev. Fluids, 1(6):064201, 2016.

37. A. R. Thornton and J.M.N.T. Gray. Breaking size segregation waves and particle recirculation in granular avalanches. J. Fluid Mech., 596:261284, 2008.

38. J.M.N.T. Gray and C. Ancey. Segregation, recirculation and deposition of coarse particles near two-dimensional avalanche fronts. J. Fluid Mech., 629:387-423, 2009.

39. J.M.N.T. Gray and B. P. Kokelaar. Large particle segregation, transport and accumulation in granular free-surface flows. J. Fluid Mech., 652: 105-137, 2010.

40. J.M.N.T. Gray and B. P. Kokelaar. Erratum large particle segregation, transport and accumulation in granular free-surface flows - erratum. J. Fluid Mech., 657:539, 2010.

41. J.C. Phillips, A.J. Hogg, R.R. Kerswell, and N.H. Thomas. Enhanced mobility of granular mixtures of fine and coarse particles. Earth and Planetary Science Letters, 246(34):466-480, 2006.

42. J.M.N.T. Gray and C. Ancey. Segregation, recirculation and deposition of coarse particles near two-dimensional avalanche fronts. J. Fluid Mech., 629:387-423, 2009.

43. B. P. Kokelaar, R. L. Graham, J.M.N.T. Gray, and J. W. Vallance. Fine-grained linings of leveed channels facilitate runout of granular flows. Earth and Planetary Science Letters, 385:172-180, 2014.

44. J. M. N. T Gray and A.R Thornton. A theory for particle size segregation in shallow granular freesurface flows. Proceedings of the Royal Society A: Mathematical, Physical and Engineering Science, 461(2057):1447-1473, 2005.

45. B. Marks, J. A. Eriksen, G. Dumazer, B. Sandnes, and K. J. Måløy. Size segregation of intruders in perpetual granular avalanches. Journal of Fluid Mechanics, 825:502-514, 2017.

46. P. Gajjar, K. van der Vaart, A. R. Thornton, C. G. Johnson, C. Ancey, and J.M.N.T. Gray. Asymmetric breaking size-segregation waves in dense granular free-surface flows. J. Fluid Mech., 794: 460-505, 2016

47. Parmesh Gajjar and J.M.N.T. Gray. Asymmetric flux models for particle-size segregation in granular avalanches. J. Fluid Mech., 757:297-329, 2014.

48. K. van der Vaart, P. Gajjar, G. Epely-Chauvin, N. Andreini, J.M.N.T. Gray, and C. Ancey. Underlying asymmetry within particle size segregation. Phys. Rev. Let., 114(23):238001, 2015.

49. L. A. Golick and K. E. Daniels. Mixing and segregation rates in sheared granular materials. Physical Review E, 80:042301, Oct 2009.

50. T. R. H. Davies. Debris-flow surges - experimental simulation. Journal of Hydrology, 29(1):18-46,
1990.

51. A. Armanini, H. Capart, L. Fraccarollo, and M. Larcher. Rheological stratification in experimental free-surface flows of granular-liquid mixtures. J. Fluid Mech., 532:269-319, 2005.

52. A. Leonardi, M. Cabrera, F. K. Wittel, R. Kaitna, M. Mendoza, W. Wu, and H. J. Herrmann. Granular-front formation in free-surface flow of concentrated suspensions. Physical Review E, 92 (5):052204, 2015.

53. P. S. Mahapatra, S. Mathew, M. V. Panchagnula, and S. Vedantam. Effect of size distribution on mixing of a polydisperse wet granular material in a belt-driven enclosure. Granular Matter, 18(2): $1-12,2016$.

54. G. Chambon, A. Ghemmour, and D. Laigle. Gravity-driven surges of a viscoplastic fluid: An experimental study. Journal of Non-Newtonian Fluid Mechanics, 158(1):54-62, 2009.

55. G. Chambon, A. Ghemmour, and M. Naaim. Experimental investigation of viscoplastic freesurface flows in a steady uniform regime. J. Fluid Mech., 754:332-364, 2014.

56. S. Wiederseiner, N. Andreini, G. Epely-Chauvin, and C. Ancey. Refractive-index and density matching in concentrated particle suspensions: a review. Experiments in Fluids, 50:1183-1206, 2011.

57. J. A. Dijksman, F. Rietz, K. A. Lörincz, M. van Hecke, and W. Losert. Invited article: Refractive index matched scanning of dense granular materials. Reviews of Scientific Instruments, 83(1): 011301, 2012

58. J. Hübl and H. Steinwendtner. Estimation of rheological properties of viscous debris flow using a belt conveyor. Physics and Chemistry of the Earth, Part B: Hydrology, Oceans and Atmosphere, 25(9):751-755, 2000.

59. T. Weinhart, R. Hartkamp, A. R. Thornton, and S. Luding. Coarse-grained local and objective continuum description of three-dimensional granular flows down an inclined surface. Phys. Fluids, 25 (7):070605, 2013.

60. D. R. Tunuguntla, A. R. Thornton, and T. Weinhart. From discrete elements to continuum fields: Extension to bidisperse systems. Comp. Part. Mech., 3(3):349-365, 2016.

61. I. Goldhirsch. Stress, stress asymmetry and couple stress: from discrete particles to continuous fields. Granular Matter, 12(3):239-252, 2010.

62. T. Weinhart, A. R. Thornton, S. Luding, and O. Bokhove. Closure relations for shallow granular flows from particle simulations. Granular Matter, 14(4):531-552, 2012.

63. G.K. Batchelor. A brief guide to two-phase flow. In Theoretical and Applied Mechanics, volume 1, pages 27-40, 1989.

64. S. C. du Pont, P. Gondret, B. Perrin, and M. Rabaud. Granular avalanches in fluids. Phys. Rev. Let., 90(4):044301, 2003.

65. M. D. Shattuck, R. A. Ingale, and P. M. Reis. Granular thermodynamics. AIP Conference Pro- 
ceedings, 1145(1):43-50, 2009

66. H. Capart, D. L. Young, and Y. Zech. Voronoi imaging methods for the measurement of granular flows. Experiments in Fluids, 32(1):121-135, 2002.

67. A. R. Thornton, T. Weinhart, V. Ogarko, and S. Luding. Multi-scale methods for multicomponent granular materials. Comp. Methods. Mat. Sc., 13(2), 2013.

68. T Weinhart, D. R. Tunuguntla, M. P. van Schrojenstein-Lantman, A. J. van der Horn, I. F. C. Denissen, C. R. Windows-Yule, A. C. de Jong, and A. R. Thornton. Mercurydpm: A fast and flexible particle solver part a: Technical advances. In Proc. 7th Int. Conf. DEM, pages 1353-1360. Springer, 2017.

69. P. A. Cundall and O. D. L. Strack. A discrete numerical model for granular assemblies. Geotechnique, 29(1):47-65, 1979

70. L. E Silbert, D. Ertaş, G. S. Grest, T. C. Halsey, D. Levine, and S. J. Plimpton. Granular flow down an inclined plane: Bagnold scaling and rheology. Physical Review E, 64(5):051302, 2001.

71. W. G. Beare and F. P. Bowden. Physical properties of surfaces. i. kinetic friction. Phil. Trans. $R$. Soc. A, 234(741):329-354, 1935.

72. T. Weinhart, A. R. Thornton, S. Luding, and O. Bokhove. From discrete particles to continuum fields near a boundary. Granular Matter, 14(2): 289-294, 2012.

73. A. R. Thornton, T. Weinhart, S. Luding., and O. Bokhove. Frictional dependence of shallowgranular flows from discrete particle simulations. European Physical Journal E, 35(12):127, 2012.

74. L. Jing, C.Y. Kwok, and Y.F. Leung. Micromechanical origin of particle size segregation. Physical Review Letters, 118(11):118001, 2017.

75. J.M.N.T. Gray and C. Ancey. Multi-component particle-size segregation in shallow granular avalanches. J. Fluid Mech., 678:535-588, 2011.

76. L. E. Silbert, J. W. Landry, and G. S. Grest. Granular flow down a rough inclined plane: transition between thin and thick piles. Physics of Fluids, 15(1):1-10, 2003.

77. J. Rajchenbach. Dense, rapid flows of inelastic grains under gravity. Phys. Rev. Let., 90(14): 144302, 2003.

78. R. A. Bagnold. Experiments on a gravity-free dispersion of large solid spheres in a newtonian fluid under shear. Proceedings of the Royal Society of London. Series A. Mathematical and Physical Sciences, 225(1160):49-63, 1954.

79. O. Hungr and S. G. Evans. Entrainment of debris in rock avalanches: an analysis of a long run-out mechanism. Geological Society of America Bulletin, 116(9-10):1240-1252, 2004.

80. B. P. Kokelaar, R. L. Graham, J.M.N.T. Gray, and J. W. Vallance. Fine-grained linings of leveed channels facilitate runout of granular flows. Earth and Planetary Science Letters, 385:172-180, 2014.

81. O. Pouliquen. Scaling laws in granular flows down rough inclined planes. Physics of Fluids, 11(3): 542-548, 1999.
82. G. Saingier, S. Deboeuf, and P.-Y. Lagrée. On the front shape of an inertial granular flow down a rough incline. Physics of Fluids, 28(5):053302, 2016.

83. L. Jing, C. Y. Kwok, Y. F. Leung, and Y. D. Sobral. Effect of geometric base roughness on size segregation. EPJ Web of Conferences, 140:03056, 2017.

84. L. Staron and J. C. Phillips. How large grains increase bulk friction in bi-disperse granular chute flows. Comp. Part. Mech., 3(3):367-372, 2016.

85. C. Goujon, N. Thomas, and B. DallozDubrujeaud. Monodisperse dry granular flows on inclined planes: Role of roughness. The European Physical Journal E, 11(2):147-157, 2003.

86. L. Jing, C. Y. Kwok, Y. F. Leung, and Y. D. Sobral. Characterization of base roughness for granular chute flows. Physical Review E, 94(5):052901, 2016.

87. Tamotsu Takahashi. Debris flow. Annual review of fluid mechanics, 13(1):57-77, 1981.

88. DE Jessop, Karim Kelfoun, Philippe Labazuy, Anne Mangeney, Olivier Roche, J-L Tillier, M Trouillet, and G Thibault. Lidar derived morphology of the 1993 lascar pyroclastic flow deposits, and implication for flow dynamics and rheology. Journal of Volcanology and Geothermal Research, 245:81-97, 2012. 